\title{
Spaces of Regular Gauge Field Configurations on a Lattice and Gauge Fixing Conditions
}

\author{
T. Bałaban * \\ Department of Physics, Harvard University, Cambridge, MA 02138, USA
}

\begin{abstract}
We consider spaces of lattice gauge field configurations satisfying gauge invariant regularity conditions, and intersections of these spaces with a surface given by gauge fixing conditions. We prove that if these conditions are chosen properly then configurations belonging to the intersection are small and regular.
\end{abstract}

In this paper we continue our investigation of the renormalization group method for lattice approximations of gauge field theories. A notion of regularity and spaces of regular gauge field configurations have appeared already in a natural way in [3]. There the compositions of averaging operations were considered and we have proved that they are regular (analytic) functions of the configurations $U$ if the following regularity conditions are satisfied

$$
|U(\partial p)-1|<\alpha_{0} \eta^{2}, \quad \eta=L^{-k}
$$

for $\alpha_{0}$ sufficiently small and for plaquettes $p$ contained in a subdomain $\Omega \subset \eta Z^{d}$. We refer the reader to $[1,3]$ for an explanation of notations and notions used here. We will use also almost all the results of those papers.

The regularity conditions ( 0.1$)$ are the most fundamental conditions we impose on gauge field configurations. They are invariant with respect to gauge transformations

$$
U\left(x, x^{\prime}\right) \rightarrow U^{u}\left(x, x^{\prime}\right)=u(x) U\left(x, x^{\prime}\right) u^{-1}\left(x^{\prime}\right),
$$

so the space of configurations satisfying $(0.1)$ is decomposed into a union of orbits determined by the group of all gauge transformations.

In this paper we will consider more complicated regularity conditions, which are gauge invariant also; but let us explain our problem and results on the example (0.1).

Our renormalization group procedure will be based on solutions of some variational problems. To consider these problems we will have to fix gauge

* Research supported in part by the National Science Foundation under Grant PHY-82-03669 
conditions, which means that we have to choose one element from each orbit, or choose a surface intersecting each orbit at exactly one element. Of course there are many such surfaces and we have to specify further our problem. We choose a configuration $U_{0}$ satisfying (0.1) and we ask if there exists a surface passing through the element $U_{0}$ and intersecting each orbit at an element sufficiently close to $U_{0}$. More precisely, this means that if $U$ is an intersection of an orbit satisfying (0.1) with the surface, then it satisfies the condition

$$
U U_{0}^{-1}=e^{i \eta A}, \quad|A|<0\left(\alpha_{0}\right),
$$

$A$ is a Lie algebra valued configuration.

This problem has a solution, i.e. there exists a surface having the above described property, but we have to introduce some further restrictions. An example of such restrictions, appearing naturally in the variational problem, is given by averaging operations

$$
\bar{U}^{k}=V \quad \text { on } \quad \Omega^{(k)}=\Omega \cap Z^{d},
$$

where $V$ is a fixed configuration on $\Omega^{(k)}$. This changes somewhat the notion of an orbit, because the conditions $(0.4)$ are invariant with respect to gauge transformations $u$ satisfying the restrictions $u=1$ on $\Omega^{(k)}$. Taking into account this change, the remaining formulations are the same.

An example of the gauge fixing conditions, having the above property, is given by the generalized Landau conditions investigated in $[1,2,4]$. In this paper we will consider these conditions only.

Actually the regularity conditions and the results are much more complicated and we refrain from the formulations in the introduction.

This paper is based on the definitions and the results of $[1,3,4]$.

\section{A. Regularity Conditions}

Let us recall the definition of a covariant derivative on a lattice. For a function $F$ defined on a subset of the lattice $T_{\eta}$, with values in complex $N \times N$ matrices, we define

$$
\begin{aligned}
& \left(D_{U, \mu}^{\eta} F\right)(x)=\eta^{-1}\left(R\left(U\left(x, x+\eta e_{\mu}\right)\right) F\left(x+\eta e_{\mu}\right)-F(x)\right), \\
& \left(D_{U, \mu}^{\eta *} F\right)(x)=\eta^{-1}\left(R\left(U\left(x, x-\eta e_{\mu}\right)\right) F\left(x-\eta e_{\mu}\right)-F(x)\right),
\end{aligned}
$$

where $U$ is an arbitrary gauge field configuration, and $R(U) X=U X U^{-1}$ for a unitary matrix $U$ and an arbitrary $X$. Let $F$ be a function defined at plaquettes of a subset of $T_{\eta}$. Let us denote by $p_{\mu v}(x)$ a plaquette determined by the point $x$ and vectors $e_{\mu}, e_{v}, \mu<v$, i.e., $p_{\mu v}(x)=\left\langle x, x+\eta e_{\mu}, x+\eta e_{\mu}+\eta e_{v}, x+\eta e_{v}\right\rangle$, and let $F_{\mu \nu}(x)=F\left(p_{\mu \nu}(x)\right)$. We define

$$
\left(D_{U}^{\eta *} F\right)\left(x, x+\eta e_{\mu}\right)=\left(D_{U}^{\eta *} F\right)_{\mu}(x)=\sum_{\nu<\mu}\left(D_{U, v}^{\eta *} F_{v \mu}\right)(x)-\sum_{\nu>\mu}\left(D_{U, v}^{\eta *} F_{\mu \nu}\right)(x) .
$$

Of course these definitions hold for an arbitrary lattice with an arbitrary scale instead of $\eta$. 
To formulate the notion of regularity we have to describe at first a geometric setting. We consider a sequence of domains (see also [2.II, 4])

$$
\Omega_{0} \supset \Omega_{1} \supset \Omega_{2} \supset \ldots \supset \Omega_{k}, \quad \Omega_{j} \subset T_{\eta}, \quad j=0,1, \ldots, k,
$$

which satisfy the following conditions:

$$
\begin{gathered}
\Omega_{j}=B^{j}\left(\Omega_{j}^{(j)}\right), \Omega_{j} \text { is a sum of cubes of a size } M_{1} L^{j} \eta, \\
\left(L^{j} \eta\right)^{-1} \operatorname{dist}\left(\Omega_{j}^{c}, \Omega_{j+1}\right)>R M_{1} .
\end{gathered}
$$

The number $M_{1}$ is a size of big blocks and was fixed in [4]. It is much bigger than $\delta_{0}^{-1}$, where $\delta_{0}$ is a decay rate of propagators, and it depends on $d$ and $L$ only. We assume that $R$ is a sufficiently large positive integer (a power of $L$ ), so that all the theorems on propagators in $[2,4]$ hold for $R M_{1}$. The sets $\Omega_{j}$ are identified with sets of bonds, or sets of plaquettes, in the following way. If $\Omega \subset T_{n}$, then we denote by $\Omega$ also the set of bonds $\bigcup_{x \in \Omega} s t(x)=\left\{\right.$ bonds $b \subset T_{\eta}$ : at least one end-point of $b$ belongs to $\Omega$ \}. Similarly for the corresponding set of plaquettes. This convention applies to an arbitrary lattice. Let us denote

$$
\Lambda_{j}=\Omega_{j}^{(j)} \backslash \Omega_{j+1}^{(j)}, \quad j=0,1, \ldots, k-1, \quad \Lambda_{k}=\Omega_{k}^{(k)},
$$

thus we have

$$
\Omega=\bigcup_{j=0}^{k} B^{j}\left(\Lambda_{j}\right), \text { where } B^{0}\left(\Lambda_{0}\right)=\Lambda_{0} .
$$

Let us notice that we admit the case where some domains $\Omega_{j}$ are equal to $T_{\eta}$, for example $\Omega_{j}=T_{\eta}$ for $j=0,1, \ldots, l, l \leqq k$.

Now we can introduce the following spaces of regular field configurations:

for a sequence (1.3) and a positive number $\alpha_{0}$ we define $\mathfrak{U}_{k}\left(\left\{\Omega_{j}\right\}, \alpha_{0}\right)$ as a set of all gauge field configurations $U$ on $T_{\eta}$ satisfying the conditions

$$
\begin{gathered}
|U(\partial p)-1|<\alpha_{0} L^{-2 j} \text { for } p \in \Omega_{j}, \quad j=0,1, \ldots, k, \\
\quad \text { or }|U(\partial p)-1|<\alpha_{0} \eta^{2}\left(L^{j} \eta\right)^{-2} \text { for } p \subset \Omega_{j}, \\
\left|\left(D_{U}^{\eta *} \partial U\right)(b)\right|<\alpha_{0} L^{-2 j}\left(L^{j} \eta\right)^{-1} \text { for } b \in \Omega_{j}, \quad j=0,1, \ldots, k .
\end{gathered}
$$

We have denoted $U(\partial p)=(\partial U)(p)$. These conditions may be written in many equivalent ways, for example (1.7) may be replaced by

$$
\eta^{-4}[1-\operatorname{Re} \operatorname{tr} U(\partial p)]<\alpha_{0}^{2}\left(L^{j} \eta\right)^{-4}, \quad p \in \Omega_{j} .
$$

Of course it is enough to assume that (1.7), (1.9) hold for $p, b \in B^{j}\left(\Lambda_{j}\right)$, because if these conditions hold for some $j=l$, then they hold for all $j<l$. These conditions are invariant with respect to gauge transformations. It is obvious for (1.7), (1.8). To see that (1.9) is invariant also let us notice that $\left(\partial U^{u}\right)\left(p_{\mu v}(x)\right)=R(u(x))(\partial U)\left(p_{\mu v}(x)\right)$, and if a function $F$ transforms as $F^{u}(x)=R(u(x)) F(x)$, then from (1.1) we get

$$
\left(D_{U^{u}, v}^{\eta *} F^{u}\right)(x)=R(u(x))\left(D_{U, v}^{\eta *} F\right)(x) .
$$

This and (1.2) imply the invariance. Thus the space $\mathfrak{H}_{k}\left(\left\{\Omega_{j}\right\}, \alpha_{0}\right)$ is invariant with respect to gauge transformations. 
Let us introduce further notations and definitions. We denote

$$
\mathfrak{B}_{k}=\bigcup_{j=0}^{k} \Lambda_{j}
$$

(the same for the sets of bonds), and for an arbitrary configuration $V$ on $\mathfrak{B}_{k}$ we define

$\mathfrak{B}_{k}\left(\mathfrak{B}_{k}, V\right)$ is a set of all gauge field configurations $U$ satisfying the conditions

$$
\bar{U}^{j}=V \quad \text { on } \quad \Lambda_{j}, \quad j=0, \ldots, k .
$$

Because $\left(\bar{U}^{u}{ }_{b}=\left(\bar{U}^{j}\right)_{b}^{u}=u\left(b_{-}\right)\left(\bar{U}^{j}\right)_{b} u^{-1}\left(b_{+}\right)\right.$for $b \subset T^{(j)}$, hence the set $\mathfrak{B}_{k}\left(\mathfrak{B}_{k}, V\right)$ is invariant with respect to gauge transformations $u$ satisfying the conditions

$$
u(y)=1 \text { for } y \in \mathfrak{B}_{k} .
$$

They form a subgroup of the group of all gauge transformations and we are interested in spaces of orbits of this subgroup.

\section{B. Gauge (Fixing) Conditions}

Generally speaking a gauge condition is a surface in a space of gauge field configurations, intersecting each orbit at exactly one point. Usually such a surface is given by equations $F(U)=0$.

There are many gauge conditions. An axial gauge is defined by the equations

$$
\begin{aligned}
& \text { for } x_{j} \in \Lambda_{j}, j=1, \ldots, k \text {, we put } \bar{U}^{j-1}\left(\Gamma_{x_{j}, x_{j-1}}\right)=1 \text { for } x_{j-1} \in B\left(x_{j}\right) \text {, } \\
& \bar{U}^{j-2}\left(\Gamma_{x_{j-1}, x_{j-2}}\right)=1 \text { for } x_{j-2} \in B\left(x_{j-1}\right), \ldots \text {, } \\
& \bar{U}\left(\Gamma_{x_{2}, x_{1}}\right)=1 \quad \text { for } \quad x_{1} \in B\left(x_{2}\right), U\left(\Gamma_{x_{1}, x}\right)=1 \quad \text { for } \quad x \in B\left(x_{1}\right) .
\end{aligned}
$$

It is easy to see that these equations together with (1.14) for gauge transformations determine uniquely an element in each orbit.

We will choose gauge conditions relative to some fixed configuration $U_{0}$. This means that if we consider new variables $U^{\prime}$ defined by

$$
U=U^{\prime} U_{0}, \quad \text { or } \quad U^{\prime}=U U_{0}^{-1},
$$

then the conditions are imposed on the variables $U^{\prime}$, and the surfaces pass through the element $U_{0}$. If we apply a gauge transformation $u$ to $U$ and if we demand that in the above representation for $U^{u}$ the configuration $U_{0}$ is unchanged, then we get the following transformation law for the configurations $U^{\prime}$ :

$$
U^{\prime u}\left(x, x^{\prime}\right)=u(x) U^{\prime}\left(x, x^{\prime}\right) R\left(U_{0}\left(x, x^{\prime}\right)\right) u^{-1}\left(x^{\prime}\right) .
$$

If we are interested in a dependence on $U_{0}$, then we may consider another point of view. We demand that $U$ and $U_{0}$ are transformed in the usual way, and then we get for ${ }^{u} U^{\prime}=U^{u}\left(U_{0}^{u}\right)^{-1}$ :

$$
{ }^{u} U^{\prime}\left(x, x^{\prime}\right)=R(u(x)) U^{\prime}\left(x, x^{\prime}\right) .
$$

Defining gauge conditions we will use the first point of view expressed in the transformation law (1.17). 
Now let us define the axial gauge conditions relative to $U_{0}$. They were introduced in [3], (64-72) by the conditions:

for $x_{0} \in B^{j}\left(x_{j}\right), x_{j} \in \Lambda_{j}, 1 \leqq j \leqq k$, we define a sequence of points $x_{0}, x_{1}, \ldots, x_{j-1}$, $x_{j}$ by the conditions $x_{n} \in B\left(x_{n+1}\right), n=0,1, \ldots, j-1$, and we put

$$
\left(\bar{R}_{0, x_{n+1}}^{n} \widetilde{U}^{\prime n}\right)\left(\Gamma_{x_{n+1}, x_{n}}\right)=\prod_{b \subset \Gamma_{x_{n+1}}, x_{n}} R\left(\bar{U}_{0}^{n}\left(\Gamma_{x_{n+1}, b-}\right)\right) \widetilde{U}_{b}^{\prime n}=1 .
$$

Let us recall that the average $\tilde{U}^{\prime n}$ was defined in [3] as

$$
\tilde{U}^{n}=\left(\overline{U^{\prime} U_{0}}\right)^{n}\left(\bar{U}_{0}^{n}\right)^{-1} .
$$

The conditions (1.19) determine uniquely an element in each orbit given by the subgroup (1.14). We denote this gauge condition by $A x_{k}\left(\mathfrak{B}_{k}, U_{0}\right)$.

Let us analyze it for $k=1$. More exactly we want to understand implications of (1.19) and (1.7). We have for a plaquette $p=\langle x, y, z, w\rangle$

$$
\begin{aligned}
U(\partial p) & =\left(U^{\prime} U_{0}\right)(\partial p) \\
& =U^{\prime}(x, y) R\left(U_{0}(x, y)\right) U^{\prime}(y, z) U_{0}(\partial p) R\left(U_{0}(x, w)\right) U^{\prime}(z, w) U^{\prime}(w, x),
\end{aligned}
$$

and let us denote

$$
\left(\partial_{U_{0}} U^{\prime}\right)(p)=U^{\prime}(x, y) R\left(U_{0}(x, y)\right) U^{\prime}(z, y) R\left(U_{0}(x, w)\right) U^{\prime}(z, w) U^{\prime}(w, x) .
$$

Let us introduce a set of bonds. For a bond $c \subset T^{(j)}$ we take a set of bonds connecting the blocks $B^{j}\left(c_{-}\right), B^{j}\left(c_{+}\right)$and denote it by $B^{j}(c)$, thus

$$
B^{j}(c)=\left\{b \subset T: b_{-} \in B^{j}\left(c_{-}\right), b_{+} \in B^{j}\left(c_{+}\right)\right\} .
$$

Later we will need to consider a general situation which is described in the lemma

Lemma 1. Let $V_{0}, V^{\prime} V_{0}$ satisfy the condition (1.7) for $k=1$ and $L$ arbitrary, and let

$$
\left(R\left(V_{0}\right) V^{\prime}\right)\left(\Gamma_{y, x}\right)=1 \quad \text { for } \quad x \in B(y),\left|\overline{V^{\prime} V_{0}}-\bar{V}_{0}\right|<\alpha_{1} \text { on } \Omega_{1}^{(1)} .
$$

Then for $\alpha_{0}, \alpha_{1}$ small the configuration $V^{\prime}$ is also small, more precisely we have the bound

$$
\left|V^{\prime}-1\right|<4 d^{2} \alpha_{0}+\alpha_{1} \quad \text { on } \Omega_{1} .
$$

We will prove the lemma. From (1.22) and the assumptions we have

$$
\left|\left(\partial_{V_{0}} V^{\prime}\right)(p)-1\right| \leqq\left|V_{0}(\partial p)-1\right|+\left|\left(V^{\prime} V_{0}\right)(\partial p)-1\right|<2 \alpha_{0} L^{-2} .
$$

The conditions $\left(R_{0} V^{\prime}\right)\left(\Gamma_{y, x}\right)=1, x \in B(y)$, imply $V_{b}^{\prime}=1$ for $b \subset \Gamma_{y, x}$. This and the above estimate imply $\left|V_{b}^{\prime}-1\right|<(d-1)(L-1) 2 \alpha_{0} L^{-2}$ for $b \subset B(y)$ by the same reasoning as in [3] (between (44) and (46)). For a plaquette $p$ connecting two neighboring blocks $B\left(c_{-}\right), B\left(c_{+}\right)$we take two bonds $b^{\prime}, b^{\prime \prime} c \partial p, b^{\prime}, b^{\prime \prime} \in B(c)$ and from (1.26) and the bounds on $\left|V_{b}^{\prime}-1\right|$ for $b^{\prime}, b^{\prime \prime}$ belonging either to $B\left(c_{-}\right)$, or to $B\left(c_{+}\right)$, we get $\left|R\left(V_{0}\left(b_{-}^{\prime}, b_{-}^{\prime \prime}\right)\right) V_{b^{\prime \prime}}^{\prime \prime}-V_{b^{\prime}}^{\prime}\right|<2 \alpha_{0} L^{-2}+4(d-1) \alpha_{0} L^{-1}<4 d \alpha_{0} L^{-1}$. Thus $\left|R\left(V_{0}\left(\Gamma_{b_{0},-, b_{-}}\right)\right) V_{b}^{\prime}-V_{b_{0}}^{\prime}\right|<(d-1)(L-1) 4 d \alpha_{0} L^{-1}<4(d-1) d \alpha_{0}$ for an arbitrary bond $b \in B(c)$ and $b_{0}$ being the unique bond of $c$ belonging to $B(c)$. The condition $\left|\bar{V}^{\prime} V_{0}-\bar{V}_{0}\right|=\left|\tilde{V}^{\prime}-1\right|<\alpha_{1}$ implies $\left|R\left(V_{0}\left(\left[c_{-}, b_{0,-}\right]\right)\right) V_{b_{0}}^{\prime}-1\right| \leqq 4(d-1)$ 
( $L-1) L \alpha_{0} L^{-2}+\alpha_{1}<4(d-1) \alpha_{0}+\alpha_{1}$, hence finally we have the bound (1.25) for an arbitrary bond $b$.

From this proof it follows that the result is local in the sense that the bound (1.25) for a bond $b$ depends on bounds (1.7), (1.24) on $B\left(c_{-}\right) \cup B\left(c_{+}\right)$, if $b$ belongs to this set.

The bound (1.25) holds for arbitrary $L$, unfortunately for $L$ large it is too weak. We expect that choosing properly a gauge condition we can get $V^{\prime}=e^{i L^{-1} A}$ with a bound $|A| \leqq O\left(\alpha_{0}+\alpha_{1}\right)$. The good gauge condition for which this is true is the Landau gauge condition considered in $[2,4]$.

Let us recall the definition of this gauge, as it was given in $[2,4]$. We consider the subspace $N\left(Q^{\prime}\left(U_{0}\right)\right)=\left\{\lambda: Q^{\prime}\left(U_{0}\right) \lambda=0\right\}$ of the space $L^{2}\left(\Omega_{0}, \mathfrak{g}\right)$ of functions on $\Omega_{0}$ with values in the Lie algebra $\mathfrak{g}$. We assume always that $U_{0}$ is a sufficiently regular configuration, so that operators depending on $U_{0}$ are well defined and all necessary properties hold for them. Later we will state precisely the assumptions. An operator $R\left(U_{0}\right)$ is defined as an orthogonal projection in this real Hilbert space, onto the subspace $\Delta_{U_{0}}^{\eta} N\left(Q^{\prime}\left(U_{0}\right)\right)$. The Landau gauge condition can be defined formally on the whole space of gauge field configurations in terms of the variables $U^{\prime}$ by the equation

$$
R\left(U_{0}\right) D_{U_{0}}^{n *} \frac{1}{i} \log U^{\prime}=0 .
$$

This definition has a sense really only for configurations in a small neighborhood of $U_{0}$, thus for $A^{\prime}=\frac{1}{i} \log U^{\prime}$ sufficiently small.

We may formulate our problem in the form of the following statement: each orbit in the space $\mathfrak{I}_{k}\left(\left\{\Omega_{j}\right\}, \alpha_{0}\right) \cap \mathfrak{B}_{k}\left(\mathfrak{B}_{k}, V\right)$ intersects the surface given by (1.27) at exactly one point $A^{\prime}$ satisfying the bounds $\left|A^{\prime}\right|<O\left(\alpha_{0} L^{-j}\right)$ on $\Omega_{j}$. Unfortunately, such a statement may not be true. Having in view also the future applications to the variational problem, we are interested in a slightly changed problem. The allowed gauge transformations $u$ are restricted by the conditions (1.14): $u(y)=1, y \in \mathfrak{B}_{k}$. These conditions are very hard to work with analytically and we have to replace them by conditions imposed on some averages of $u$ rather than on values of $u$ at the points of $\mathfrak{B}_{k}$. We choose the averages which were described in detail in [3], formulas (78)-(80), and denoted by $\bar{R}_{0} u^{j}$. Let us notice that for $u(x)=e^{i \lambda(x)}$ and $\lambda$ small a linear part of the function $\frac{1}{i} \log \left(\overline{R_{0} u^{j}}\right)(y)$ is equal to $\left(Q_{j}^{\prime}\left(U_{0}\right) \lambda\right)(y)$, and the definition of the Landau gauge depends on these averaging operations. The restrictions on gauge transformations are now given by the conditions $\left(\overline{R_{0} u^{j}}\right)(y)=1, y \in \Lambda_{j}, j=0,1, \ldots, k$. The problem with these conditions is that they do not agree with the group structure of the space of gauge transformations, that is if $u_{1}, u_{2}$ are two transformations satisfying these conditions, then generally the product $u_{1} u_{2}$ does not satisfy them. This implies that the notion of orbit is not well defined and depends on an element $U$ chosen to generate the set $\left\{U^{u^{-1}}\right\}$. Let us remark that we have changed the convention how to define an orbit. From now on we will use inverse elements $u^{-1}$, in accordance with the definitions and formulas of the paper [3]. A way out of this difficulty is to choose in some way an element $U$ 
from each orbit defined by (1.14), that is to choose some preliminary gauge condition, next to generate the set $\left\{U^{u^{-1}}\right\}$ for all $u$ satisfying the conditions ${\overline{R_{0}}}^{j}=1$, and finally to find an intersection of this set with the surface determined by the Eq. (1.27). We would like to prove that this intersection is unique and that the corresponding configuration $A$ satisfies the bounds $|A|<O\left(\alpha_{0} L^{-j}\right)$ on $\Omega_{j}$.

As the preliminary gauge condition we choose the axial gauge given by the Eq. (1.19). Now we can formulate the problem precisely. Let us take an arbitrary $U^{\prime}$ such that

$$
U^{\prime} U_{0} \in \mathfrak{U}_{k}\left(\left\{\Omega_{j}\right\}, \alpha_{0}\right) \cap \mathfrak{B}_{k}\left(\mathfrak{B}_{k}, V\right) \cap A x_{k}\left(\mathfrak{B}_{k}, U_{0}\right)
$$

and let us consider the set $\left\{U^{\prime u^{-1}}\right\}$ for gauge transformations $u$ satisfying the conditions

$$
\left({\overline{R_{0}}}^{j}\right)(y)=1 \quad \text { for } \quad y \in \Lambda_{j}, \quad j=0,1, \ldots, k .
$$

Our problem is to prove that there exists a gauge transformation $u$ such that $U^{\prime u^{-1}}$, or rather $A=\frac{1}{i} \log U^{u^{-1}}$, satisfies the Landau gauge condition (1.27) and the bounds $|A|,\left|\nabla_{U_{0}}^{L-j} A\right|<O\left(\alpha_{0} L^{-j}\right)$ on $\Omega_{j}$. We would like to prove also that such a gauge transformation is unique, i.e. the set $\left\{U^{\prime u^{-1}}\right\}$ intersects the part of the surface (1.27) satisfying the above bounds at exactly one point.

The rest of this paper is devoted to a proof of this statement.

Let us make few remarks assuming that this statement is true. The unique gauge transformations described in it define a mapping of the set (1.28) into the regular part of the surface (1.27). Let us denote the image set of this mapping by $\sum_{k}$, and $U^{u^{-1}}=U_{1}$. The set $\sum_{k}$ is not contained in $\mathfrak{B}_{k}\left(\mathfrak{B}_{k}, V\right)$ because the gauge transformations $u$ do not satisfy the necessary conditions (1.14). The configurations $U^{\prime}$ satisfy the equations $\tilde{U}^{\prime j}=V\left(\bar{U}_{0}^{j}\right)^{-1}$ on $\Lambda_{j}$, hence $U_{1}$ satisfies

$$
\left(\tilde{U}_{1}^{u j}\right)_{b}=u\left(b_{-}\right)\left(\widetilde{U}_{1}^{j}\right)_{b} \bar{R}_{0, b}^{j} u^{-1}\left(b_{+}\right)=V_{b}\left(\bar{U}_{0}^{j}\right)_{b}^{-1}, b \in \Lambda_{j} .
$$

Let us notice that we do not assume that $U_{0}$ satisfies (1.13), i.e. that $U_{0} \in \mathfrak{B}_{k}\left(\mathfrak{B}_{k}, V\right)$, hence the right-hand side in (1.30) generally is not equal to 1 . We will assume only that it is close to 1 .

In [3] we have determined the gauge transformation $u$ in terms of the configuration $U_{1}$. We refer the reader especially to the formulas (87), (99), (97), and (105) of that paper. If both end-points $b_{-}, b_{+}$of a bond $b$ belong to $\Lambda_{j}$, then the expression on the left-hand side of $(1.30)$ is equal to $\left(\bar{U}_{1}^{j}\right)_{b}$ by (92) of [3]. If a bond $b$ crosses the boundary of $\Lambda_{j}$, then one of the end-points belongs to $\Lambda_{j}$, e.g. $b_{+} \in \Lambda_{j}$, and another to $\Lambda_{j-1}, b_{-} \in \Lambda_{j-1}$. In this case the formulas (97), (99), and (87) of [3] imply

$$
\left(\widetilde{U}_{1}^{u j}\right)_{b}=\overline{\bar{R}_{0, b-}^{j-1} \bar{U}_{1}^{j-1}}\left(\bar{U}_{1}^{j}\right)_{b}=V_{b}\left(\bar{U}_{0}^{j}\right)_{b}^{-1},
$$

where by the formula (105) of [3]

$$
\overline{\bar{R}_{0, b-}^{j-1} \bar{U}_{1}^{j-1}}=\exp \left[i \sum_{x \in B\left(b_{-}\right)} L^{-d} \frac{1}{i} \log \left(\bar{R}_{0, b_{-}}^{j-1} \bar{U}_{1}^{j-1}\right)\left(\Gamma_{b_{-}, x}\right)\right] .
$$


All sites of the contours $\Gamma_{b_{-}, x}$ belong to $\Lambda_{j-1}$, hence $\bar{U}_{1}^{j-1}$ in the above formula is equal to $V\left(\bar{U}_{0}^{j-1}\right)^{-1}$. Let us denote $V\left(\bar{U}_{0}^{j}\right)^{-1}=V^{\prime}$. We can write Eq. (1.30) as

or

$$
\begin{aligned}
\left(\bar{U}_{1}^{j}\right)_{b} & =V_{b}^{\prime}=\exp i B_{b}, \quad \text { if } \quad b \subset \Lambda_{j}\left(\text { i.e., } b_{-}, b_{+} \in \Lambda_{j}\right), \\
\left(\bar{U}_{1}^{j}\right)_{b} & =\exp \left[-i \sum_{x \in B(b-)} L^{-d} \frac{1}{i} \log \left(\bar{R}_{0, b-}^{j-1} V^{\prime}\right)\left(\Gamma_{b_{-}, x}\right)\right] V_{b}^{\prime} \\
& =\exp i B_{b}, \quad \text { if } b_{-} \in \Lambda_{j-1}, b_{+} \in \Lambda_{j},
\end{aligned}
$$

$$
\frac{1}{i} \log \bar{U}_{1}^{j}=B \quad \text { on } \quad \Lambda_{j}, \quad j=0,1, \ldots, k,
$$

where the configuration $B$ is defined by the above equations. Thus we have

$\sum_{k} \subset\left\{\right.$ a set of configurations $U=U_{1} U_{0}, U \in \mathfrak{H}_{k}\left(\left\{\Omega_{j}\right\}, \alpha_{0}\right), U_{1}=e^{i \eta A}$,

$$
\begin{aligned}
& |A|<O\left(\alpha_{0}\left(L^{j} \eta\right)^{-1}\right),\left|\nabla_{U_{0}}^{\eta} A\right|<O\left(\alpha_{0}\left(L^{j} \eta\right)^{-2}\right) \quad \text { on } \Omega_{j}, Q\left(U_{0}, \eta A\right)=B, \\
& \left.R\left(U_{0}\right) D_{U_{0}}^{\eta *} A=0\right\} .
\end{aligned}
$$

Let us remark also that we have chosen the preliminary gauge condition $A x_{k}$ not only for the reason of simplicity and convenience, but for deeper reasons connected with properties of the averaging operations $\bar{U}_{1}^{j}$.

\section{Formulation of Theorems on Transformation from the Axial Gauge to the Landau Gauge}

At first let us formulate precisely the assumptions we have to make on the configurations $U_{0}, U=U^{\prime} U_{0}$. We assume that:

$$
\begin{gathered}
U_{0} \in \mathfrak{U}_{k}\left(\left\{\Omega_{j}\right\}, \alpha_{0}\right), \quad U_{0} \text { satisfies the regularity condition (3.35) in [4] } \\
\qquad U^{\prime} U_{0} \in \mathfrak{U}_{k}\left(\left\{\Omega_{j}\right\}, \alpha_{0}\right) \cap A x_{k}\left(\mathfrak{B}_{k}, U_{0}\right) \\
\left|\left(\overline{U^{\prime} U_{0}}\right)^{j}-\bar{U}_{0}^{j}\right|<\alpha_{1} \quad \text { on } \Lambda_{j}, \quad j=0,1, \ldots, k
\end{gathered}
$$

We need the second condition in (1.33) to apply all the results of [4] on propagators with the background gauge field configuration $U_{0}$. We will see later that this condition is a consequence of the first one in (1.33), so eventually we will drop it out of the assumptions. The condition (1.35) replaces the equalities for the averages in the conditions (1.28). It is satisfied if $V$ is close to $\bar{U}_{0}^{j}$, more precisely if $\left|V-\bar{U}_{0}^{j}\right|<\alpha_{1}$.

Now our problem is to construct a gauge transformation $u$ satisfying the equalities (1.29) and such that $U_{1}=U^{\prime u^{-1}}$ satisfies the conditions

$$
\begin{gathered}
U_{1}=e^{i \eta A},|A|<B_{1}\left(\alpha_{0}+\alpha_{1}\right)\left(L^{j} \eta\right)^{-1},\left|\nabla_{U_{0}}^{\eta} A\right|<B_{1}\left(\alpha_{0}+\alpha_{1}\right)\left(L^{j} \eta\right)^{-2}, \\
\|A\|_{1, \beta}<B_{2}\left(\beta_{0}\right)\left(\alpha_{0}+\alpha_{1}\right)\left(L^{j} \eta\right)^{-2-\beta}, \beta \leqq \beta_{0}<1, \quad \text { on } \quad \Omega_{j}, \quad j=0,1, \ldots, k,
\end{gathered}
$$

$Q_{j}\left(U_{0}, \eta A\right)=B$ on $\Lambda_{j}, \quad j=0,1, \ldots, k, B$ is given by formula (1.31) with

$$
V^{\prime}=\widetilde{U}^{\prime j} \text {, }
$$

$|B|<2 d L \alpha_{1}$ by the assumption (1.35),

$$
R\left(U_{0}\right) D_{U_{0}}^{\eta *} A=0 .
$$


Of course the last condition is the most important constraint on $A$ which assures that the others are satisfied. It holds by the construction. Also the condition (1.37) is basically of an algebraic character and it follows from the construction, as in (1.30), (1.31). The only fact we need to write it this way is the representation of $U_{1}$ and the first condition on $A$ in (1.36). Thus (1.36) contains all the results we have to prove about $A$, besides its existence. This condition describes fully regularity properties of the first order derivatives. Such information is unavailable for the second order derivatives, but we have the following bounds for the second order operators acting on $A$ :

$$
\left|D_{U_{0}}^{\eta *} D_{U_{0}}^{\eta} A\right|,\left|\Delta_{U_{0}}^{\eta} A\right|<B_{1}\left(\alpha_{0}+\alpha_{1}\right)\left(L^{j} \eta\right)^{-3} \quad \text { on } \quad \Omega_{j}, \quad j=0,1, \ldots, k .
$$

Of course we want to prove that the constants $B_{1}, B_{2}\left(\beta_{0}\right)$ in (1.36), (1.39) are absolute constants depending on $d$ and $L$ only, $B_{2}\left(\beta_{0}\right)$ on $\beta_{0}$ also. We expect that we have to assume that $\alpha_{0}+\alpha_{1}$ is sufficiently small.

We can formulate now the main result of the paper.

Theorem 2. There exist constants $B_{1}, B_{2}\left(\beta_{0}\right), c_{1}$ such that for arbitrary $U_{0}, U^{\prime} U_{0}$ satisfying (1.33)-(1.35) with $\alpha_{0}+\alpha_{1} \leqq c_{1}$ there exists exactly one gauge transformation $u$ satisfying (1.29) and such that the conditions (1.36)-(1.39) hold for the configuration $U_{1}=U^{\prime u^{-1}}$.

We will be proving this theorem, and its reformulations, in the rest of this paper.

Let us separate some aspects of the proof. It will be by induction with respect to $k$ and we will construct the configuration $U_{1}$ perturbatively, the perturbation around a configuration constructed in a previous step. This method will not give us good constants in (1.36), or (1.39), and we have to prove the bounds in (1.36), (1.39) by some other method, assuming that we have constructed $U_{1}$ satisfying (1.36)-(1.39) with worse constants. This raises the question of what are the best constants in these bounds. This question may be answered independently of a proof of Theorem 2 .

Let us consider a somewhat more general situation. We assume that we have configurations $U_{0}, U_{1} U_{0}$ satisfying the following conditions

$$
\begin{aligned}
& U_{0}, U_{1} U_{0} \in \mathfrak{U}_{k}\left(\left\{\Omega_{j}\right\}, \alpha_{0}\right), U_{0} \\
& \text { satisfies the additional regularity condition }(3.35) \text { in }[4], \\
& \qquad U_{1}=e^{i \eta A},|A|<\alpha_{2}\left(L^{j} \eta\right)^{-1} \text { on } \Omega_{j}, \\
& R\left(U_{0}\right) D_{U_{0}}^{\eta *} A=0, Q_{j}\left(U_{0}, \eta A\right)=B \text { on } \Lambda_{j}, \quad|B|<2 d L \alpha_{1},
\end{aligned}
$$

$(j=0,1, \ldots, k$ above). Thus we have apparently much weaker regularity now. We will prove that in fact these conditions imply all the other conditions in (1.36), (1.39), and we will find the constants $B_{1}, B_{2}$, and restrictions on $\alpha_{0}, \alpha_{1}, \alpha_{2}$. We start investigating implications of (1.40).

We derive formulas and estimates which will have other applications in the future, so we formulate them now in the most general way needed. We have from (1.21), (1.22),

$$
\begin{aligned}
\left(U_{1} U_{0}\right)(\partial p)-1= & {\left[U_{1}(x, y) R\left(U_{0}(x, y)\right) U_{1}(y, z)-1\right]\left[U_{0}(\partial p)-1\right] } \\
& \cdot R\left(U_{0}(x, w)\right) U_{1}(z, w) U_{1}(w, x) \\
& +\left[U_{0}(\partial p)-1\right]\left[R\left(U_{0}(x, w)\right) U_{1}(z, w) U_{1}(w, x)-1\right] \\
& +\left[U_{0}(\partial p)-1\right]+\left[\left(\partial_{U_{0}} U_{1}\right)(p)-1\right] .
\end{aligned}
$$


The first two terms on the right-hand side can be bounded by $4 \alpha_{2} \alpha_{0}\left(L^{j} \eta\right)^{-3} \eta^{3}$ for $p$ $C \Omega_{j}$. If we apply the derivative $D_{U_{1} U_{0}}^{\eta *}$ to $\partial U_{1} U_{0}$, then the derivative of these two terms can be bounded by $8 d \alpha_{2} \alpha_{0}\left(L^{j} \eta\right)^{-3} \eta^{2}$. Further we have

$$
\left|D_{U_{1} U_{0}}^{\eta *} \partial U_{0}-D_{U_{0}}^{\eta *} \partial U_{0}\right|<2 d \alpha_{0} \alpha_{2}\left(L^{j} \eta\right)^{-3} \eta^{2} \text { on } \Omega_{j} .
$$

Let us denote by $O_{1}(\alpha)$ an expression which can be estimated by $\alpha$, i.e. $\left|O_{1}(\alpha)\right| \leqq \alpha$. Thus we have

$$
D_{U_{1} U_{0}}^{\eta *} \partial U_{1} U_{0}=D_{U_{0}}^{\eta *} \partial U_{0}+D_{U_{1} U_{0}}^{\eta *} \partial_{U_{0}} U_{1}+O_{1}\left(10 d \alpha_{0} \alpha_{2}\left(L^{j} \eta\right)^{-3} \eta^{2}\right) .
$$

Let us consider the second term on the right-hand side above. From the definition (1.1), (1.2) we obtain that a value of this expression at a bond $\left\langle x, x+\eta e_{\mu}\right\rangle \in \Omega_{j}$ is equal to

$$
\begin{aligned}
& \left(D_{U_{1} U_{0}}^{n *} \partial_{U_{0}} U_{1}\right)\left(x, x+\eta e_{\mu}\right)=\left(D_{U_{1} U_{0}}^{n *}\left(\partial_{U_{0}} U_{1}-1\right)\right)\left(x, x+\eta e_{\mu}\right) \\
& \quad=\left(D_{U_{0}}^{\eta *}\left(\partial_{U_{0}} U_{1}-1\right)\right)\left(x, x+\eta e_{\mu}\right) \\
& \quad+\sum_{v<\mu} \eta^{-1} R\left(U_{0}\left(x, x-\eta e_{v}\right)\right) \\
& \quad \cdot\left(R\left(U_{1}\left(x, x-\eta e_{v}\right)\right)-1\right)\left(\left(\partial_{U 0} U_{1}\right)\left(p_{v \mu}\left(x-\eta e_{v}\right)\right)-1\right) \\
& \quad-\sum_{v>\mu} \eta^{-1} R\left(U_{0}\left(x, x-\eta e_{v}\right)\right)\left(R\left(U_{1}\left(x, x-\eta e_{v}\right)\right)-1\right)\left(\left(\partial_{U_{0}} U_{1}\right)\left(p_{\mu v}\left(x-\eta e_{v}\right)\right)-1\right) .
\end{aligned}
$$

We expand $R\left(U_{1}\left(x, x-\eta e_{v}\right)\right)-1$ in $A$. Because $\partial_{U_{0}} U_{1}-1=O_{1}\left(4 \alpha_{2}\left(L^{j} \eta\right)^{-1} \eta\right)$, so it is enough to consider a term of the first order, a remainder is $O_{1}\left(3|\eta A|^{2}\right)$ $=O_{1}\left(3 \alpha_{2}^{2}\left(L^{j} \eta\right)^{-2} \eta^{2}\right)$. The term of the first order is $i \eta \operatorname{ad}_{A\left(x, x-\eta e_{v}\right)}=O_{1}\left(2 \alpha_{2}\left(L^{j} \eta\right)^{-1} \eta\right)$. Similarly, if we take this term and expand $\partial_{U_{0}} U_{1}-1$, then it is enough to consider a term of the first order only, a remainder is $O_{1}\left(\frac{1}{2 !} \eta^{2}(\partial|A|(p))^{2}\right)$ $=O_{1}\left(8 \alpha_{2}^{2}\left(L^{j} \eta\right)^{-2} \eta^{2}\right)$. The term of the first order is $i \eta^{2}\left(D_{U_{0}}^{\eta} A\right)(p)$. This gives

$$
\begin{aligned}
& \left(D_{U_{1} U_{0}}^{\eta *} \partial_{U_{0}} U_{1}\right)\left(x, x+\eta e_{\mu}\right)=\left(D_{U_{0}}^{\eta *}\left(\partial_{U_{0}} U_{1}-1\right)\right)\left(x, x+\eta e_{\mu}\right) \\
& \quad+\eta^{2} \sum_{v \neq \mu} R\left(U_{0}\left(x, x-\eta e_{v}\right)\right) \operatorname{ad}_{A\left(x, x-\eta e_{v}\right)}\left(D_{U_{0}}^{\eta} A\right)\left(p_{\mu v}\left(x-\eta e_{v}\right)\right) \\
& \quad+O_{1}\left(28 d \alpha_{2}^{3}\left(L^{j} \eta\right)^{-3} \eta^{2}\right) .
\end{aligned}
$$

Let us consider the first term on the right-hand side of the above equality. We expand it in $A$ up to the third order and we get

$$
\begin{aligned}
\left(\partial_{U_{0}} U_{1}\right)(p)-1= & i \eta^{2}\left(D_{U_{0}}^{\eta} A\right)(p)-\frac{1}{2} \eta^{2} V_{2}\left(U_{0}, A, \partial p\right) \\
& +O_{1}\left(\frac{1}{3 !} \eta^{3}(\partial|A|(p))^{3}\right),
\end{aligned}
$$

where $V_{2}$ is a polynomial of the second order in $A$. Thus

$$
D_{U_{0}}^{\eta *}\left(\partial_{U_{0}} U_{1}-1\right)=i \eta^{2} D_{U_{0}}^{\eta *} D_{U_{0}}^{\eta} A-\frac{1}{2} \eta^{2} D_{U_{0}}^{\eta *} V_{2}\left(U_{0}, A\right)+O_{1}\left(\frac{4^{3}}{3} d \alpha_{2}^{3}\left(L^{j} \eta\right)^{-3} \eta^{2}\right) .
$$


We find easily the polynomial $V_{2}$ :

$$
\begin{aligned}
V_{2}\left(U_{0}, A, \partial p\right)= & (A(x, y))^{2}+2 A(x, y) R\left(U_{0}(x, y)\right) A(y, z) \\
& +2 A(x, y) R\left(U_{0}(x, w)\right) A(z, w) \\
& +2 A(x, y) A(w, x)+\left(R\left(U_{0}(x, y)\right) A(y, z)\right)^{2} \\
& +2 R\left(U_{0}(x, y)\right) A(y, z) R\left(U_{0}(x, w)\right) A(z, w) \\
& +2 R\left(U_{0}(x, y)\right) A(y, z) A(w, x) \\
& +\left(R\left(U_{0}(x, w)\right) A(z, w)\right)^{2} \\
& +2 R\left(U_{0}(x, w)\right) A(z, w) A(w, x)+(A(w, x))^{2} \\
= & \eta^{2}\left(\left(D_{U_{0}}^{\eta} A\right)(p)\right)^{2}+\left\{\left[A(x, y), R\left(U_{0}(x, y)\right) A(y, z)\right]\right. \\
& +\left[A(x, y), R\left(U_{0}(x, w)\right) A(z, w)\right]+[A(x, y), A(w, x)] \\
& +\left[R\left(U_{0}(x, y)\right) A(y, z), R\left(U_{0}(x, w)\right) A(z, w)\right] \\
& +\left[R\left(U_{0}(x, y)\right) A(y, z), A(w, x)\right] \\
& \left.+\left[R\left(U_{0}(x, w)\right) A(z, w), A(w, x)\right]\right\} .
\end{aligned}
$$

This equality holds for arbitrary $A$ in the complexified Lie algebra, but if $A$ is Hermitian, then the first expression above is a real part of $V_{2}$, and the second is an imaginary part of $V_{2}$, multiplied by $i$. Let $p=p_{\mu \nu}(x)$, then the expression in $\{\ldots\}$ can be written as

$$
\begin{aligned}
\{\ldots\}= & 2\left[A_{\mu}(x), A_{v}(x)\right]+2 \eta\left[A_{\mu}(x),\left(D_{\mu} A_{v}\right)(x)\right] \\
& +2 \eta\left[\left(D_{v} A_{\mu}\right)(x), A_{v}(x)\right]-\eta\left[A_{\mu}(x),\left(D_{v} A_{\mu}\right)(x)\right] \\
& -\eta\left[\left(D_{\mu} A_{v}\right)(x), A_{v}(x)\right]-\eta^{2}\left[\left(D_{\mu} A_{v}\right)(x),\left(D_{v} A_{\mu}\right)(x)\right],
\end{aligned}
$$

where $D_{\mu}$ denotes the derivative $D_{U_{0}, \mu}^{\eta}$. From these equalities we see that all the terms in $V_{2}$, except the first term on the right-hand side above, have some powers of $\eta$. If we apply $D_{U_{0}}^{\eta *}$ to $V_{2}$, we use these factors to cancel $\eta^{-1}$ and to replace $D_{U_{0}}^{n *}$ by $D_{U_{0}}^{1 *}$, which is a very simple bounded operator. Thus

$$
D_{U_{0}}^{n *} V_{2}\left(U_{0}, A\right)=-2 \sum_{v=1}^{d} D_{v}^{*}\left[A_{\mu}, A_{v}\right]+O_{1}\left(48 d|A|\left|\nabla_{U_{0}}^{\eta} A\right|\right) .
$$

Further

$$
\begin{aligned}
\left(D_{v}^{*}\left[A_{\mu}, A_{v}\right]\right)(x)= & \eta\left[\left(D_{v}^{*} A_{\mu}\right)(x),\left(D_{v}^{*} A_{v}\right)(x)\right]+\left[\left(D_{v}^{*} A_{\mu}\right)(x), A_{v}(x)\right] \\
& +\left[A_{\mu}(x),\left(D_{v}^{*} A_{v}\right)(x)\right]
\end{aligned}
$$

hence, taking into account the identity $\left(D_{\mu}^{*} A\right)(x)=-R\left(U_{0}\left(x-\eta e_{\mu}, x\right)\right)\left(D_{\mu} A\right)$ $\left(x-\eta e_{\mu}\right)$, we get

$$
\frac{1}{2} \eta^{2} D_{U_{0}}^{\eta *} V_{2}\left(U_{0}, A\right)=O_{1}\left(32 d|A|\left|\nabla_{U_{0}}^{\eta} A\right| \eta^{2}\right)=O_{1}\left(32 d \alpha_{2}\left(L^{j} \eta\right)^{-1}\left|\nabla_{U_{0}}^{\eta} A\right| \eta^{2}\right) .
$$

Gathering together the equalities and the estimates obtained until now we get

$$
\begin{aligned}
D_{U_{1} U_{0}}^{\eta *} \partial U_{1} U_{0}= & D_{U_{0}}^{\eta *} \partial U_{0}+i \eta^{2} D_{U_{0}}^{\eta *} D_{U_{0}}^{\eta} A+O_{1}\left(36 d \alpha_{2}\left(L^{j} \eta\right)^{-1}\left|\nabla_{U_{0}}^{\eta} A\right| \eta^{2}\right) \\
& +O_{1}\left(50 d \alpha_{2}^{3}\left(L^{j} \eta\right)^{-3} \eta^{2}\right)+O_{1}\left(10 d \alpha_{0} \alpha_{2}\left(L^{j} \eta\right)^{-3} \eta^{2}\right) \text { on } \Omega_{j} .
\end{aligned}
$$


This is the basic estimate. It will be applied in several different situations in the future. Let us recall that it was derived under the assumption (0.1) for $U_{0}$ and (1.41) for $U_{1}$.

We will apply it to get regularity results for $A$, assuming (1.40)-(1.42). From (1.40) the term on the left-hand side and the first term on the right-hand side can be estimated by $\alpha_{0}\left(L^{j} \eta\right)^{-3} \eta^{2}$. This implies that

$$
\begin{gathered}
D_{U_{0}}^{\eta *} D_{U_{0}}^{\eta} A=J, \\
|J| \leqq\left(2 \alpha_{0}+36 d \alpha_{2}\left(L^{j} \eta\right)^{2}\left|\nabla_{U_{0}}^{\eta} A\right|+50 d \alpha_{2}^{3}+10 d \alpha_{0} \alpha_{2}\right)\left(L^{j} \eta\right)^{-3},
\end{gathered}
$$

or

$$
|J|_{(-3)} \leqq 2 \alpha_{0}+36 d \alpha_{2}\left|\nabla_{U_{0}}^{\eta} A\right|_{(-2)}+50 d \alpha_{2}^{3}+10 d \alpha_{0} \alpha_{2},
$$

where the norms $|\cdot|_{(\alpha)}$ were introduced in [4]. For the reader's convenience let us recall the definition: $|A|_{(\alpha)}=\sup _{j} \sup _{\Omega_{j}}\left(L^{j} \eta\right)^{-\alpha}|A|$.

The configuration $A$ satisfies (1.42) also. Proposition 4 from [3] implies that

$$
Q_{j}\left(U_{0}, \eta A\right)=L^{j} \eta Q_{j} A+C_{j}\left(L^{j} \eta A\right),\left|C_{j}\left(L^{j} \eta A\right)\right| \leqq C_{2}\left|L^{j} \eta A\right|^{2}<C_{2} \alpha_{2}^{2},
$$

hence $L^{j} \eta Q_{j} A=B_{1}, B_{1}=B-C_{j}\left(L^{j} \eta A\right)$ on $\Lambda_{j},\left|B_{1}\right|<2 d L \alpha_{1}+C_{2} \alpha_{2}^{2}$. We make the translation $A=A_{1}+H\left(U_{0}\right) B_{1}$, where the operator $H\left(U_{0}\right)$ was defined in [4], and we get the equations

$$
\begin{gathered}
D_{U_{0}}^{\eta *} D_{U_{0}}^{\eta} A_{1}=J-D_{U_{0}}^{\eta *} D_{U_{0}}^{\eta} H\left(U_{0}\right) B_{1}, \\
Q_{j} A_{1}=0 \quad \text { on } \quad \Lambda_{j}, \quad R\left(U_{0}\right) D_{U_{0}}^{\eta *} A_{1}=0 .
\end{gathered}
$$

They imply finally

$$
\begin{aligned}
A & =G\left(U_{0}\right) J-G\left(U_{0}\right) D_{U_{0}}^{\eta *} D_{U_{0}} H\left(U_{0}\right) B_{1}+H\left(U_{0}\right) B_{1} \\
& =G\left(U_{0}\right) J+G\left(U_{0}\right) \sum_{j} Q_{j}^{*} \Lambda_{j}\left(L^{j} \eta\right)^{-3} B_{1},
\end{aligned}
$$

where the operator $G\left(U_{0}\right)$ was introduced and investigated in [4]. Let us recall only the definition:

$$
G\left(U_{0}\right)=\left(D_{U_{0}}^{n *} D_{U_{0}}^{\eta}+D_{U_{0}}^{\eta} R\left(U_{0}\right) D_{U_{0}}^{n *}+\sum_{j} Q_{j}^{*} \Lambda_{j}\left(L^{j} \eta\right)^{-2} Q_{j}\right)^{-1} .
$$

Theorem 3.3 of [4] implies the bounds:

$$
\begin{aligned}
& |A|_{(-1)},\left|V_{U_{0}}^{\eta} A\right|_{(-2)},\left|D_{U_{0}}^{\eta *} D_{U_{0}}^{\eta} A\right|_{(-3)},\left|\Delta_{U_{0}}^{\eta} A\right|_{(-3)} \leqq B_{0}\left(|J|_{(-3)}+\left|B_{1}\right|\right) \\
& \quad \leqq B_{0}\left(2 \alpha_{0}+36 d \alpha_{2}\left|\nabla_{U_{0}}^{\eta} A\right|_{(-2)}+50 d \alpha_{2}^{3}+10 d \alpha_{0} \alpha_{2}+2 d L \alpha_{1}+C_{2} \alpha_{2}^{2}\right) .
\end{aligned}
$$

Let us take this bound for $\left|\nabla_{U_{0}}^{\eta} A\right|_{(-2)}$ on the left-hand side, and let us assume that $B_{0} 36 d \alpha_{2} \leqq 1 / 2$. This gives us a bound for $\left|\nabla_{U_{0}}^{\eta} A\right|_{(-2)}$, equal to the right-hand side above without this term and multiplied by 2 . Using this bound we get

$$
\begin{aligned}
& |A|_{(-1)},\left|\nabla_{U_{0}}^{\eta} A\right|_{(-2)},\left|D_{U_{0}}^{\eta *} D_{U_{0}}^{\eta} A\right|_{(-3)},\left|\Delta_{U_{0}}^{\eta} A\right|_{(-3)} \\
& \quad<B_{0}\left(4 \alpha_{0}+4 d L \alpha_{1}+2 \alpha_{2}^{2}+20 d \alpha_{0} \alpha_{2}+2 C_{2} \alpha_{2}^{2}\right)
\end{aligned}
$$

Now we assume further that

$$
2 \alpha_{2}^{2}+20 d \alpha_{0} \alpha_{2}+2 C_{2} \alpha_{2}^{2} \leqq \alpha_{0}+\alpha_{1} .
$$


This and the previous inequality give finally

$$
\begin{aligned}
|A| & <5 d L B_{0}\left(\alpha_{0}+\alpha_{1}\right)\left(L^{j} \eta\right)^{-1},\left|\nabla_{U_{0}}^{\eta} A\right|<5 d L B_{0}\left(\alpha_{0}+\alpha_{1}\right)\left(L^{j} \eta\right)^{-2}, \\
\|A\|_{1, \beta} & <5 d L B_{0}(\beta)\left(\alpha_{0}+\alpha_{1}\right)\left(L^{j} \eta\right)^{-2-\beta},\left|D_{U_{0}}^{\eta *} D_{U_{0}}^{\eta} A\right|,\left|\Delta_{U_{0}}^{\eta} A\right| \\
& <5 d L B_{0}\left(\alpha_{0}+\alpha_{1}\right)\left(L^{j} \eta\right)^{-3} \text { on } \Omega_{j} .
\end{aligned}
$$

Let us formulate the results in

Proposition 3. If $U_{0}, U_{1} U_{0}$ satisfy (1.40)-(1.42) with $\alpha_{0}, \alpha_{1}, \alpha_{2}$ bounded by a constant depending on $d$ and Lonly, and $\alpha_{2}$ satisfies the additional restriction (1.61), then $U_{1}$ satisfies (1.36)-(1.39) with $B_{1}=5 d L B_{0}, B_{2}\left(\beta_{0}\right)=5 d L B_{0}\left(\beta_{0}\right)$, where $B_{0}$, $B_{0}\left(\beta_{0}\right)$ are the corresponding norms of the operators $G\left(U_{0}\right), H\left(U_{0}\right)$, and depend ond and $L$ only, $B_{0}\left(\beta_{0}\right)$ on $\beta_{0}$ also.

This proposition explains how the constants $B_{1}, B_{2}$ may be chosen. Let us make some comments on the restriction (1.61). In the future we will get a configuration $A$ satisfying (1.41) with $\alpha_{2}=B_{1}^{\prime}\left(\alpha_{0}+\alpha_{1}\right)=C_{1}^{\prime} B_{1}\left(\alpha_{0}+\alpha_{1}\right)$. The restriction follows from the bounds

$$
20 d \alpha_{2} \alpha_{0}+2 \alpha_{2}^{2}+2 C_{2} \alpha_{2}^{2}<\left(20 d B_{1} C_{1}^{\prime}+2 B_{1}^{2} C_{1}^{\prime 2}+2 C_{2} B_{1}^{2} C_{1}^{\prime 2}\right)\left(\alpha_{0}+\alpha_{1}\right)^{2} \leqq \alpha_{0}+\alpha_{1},
$$

hence from

$$
\left(20 d B_{1} C_{1}^{\prime}+2\left(1+C_{2}\right) B_{1}^{2} C_{1}^{\prime 2}\right)\left(\alpha_{0}+\alpha_{1}\right) \leqq 1 .
$$

This is a restriction on $\alpha_{0}+\alpha_{1}$ depending on $d, L$, and $C_{1}^{\prime}$, and in the future considerations we will have to make sure that $C_{1}^{\prime}$ depends on $d$ and $L$ only.

We will prove Theorem 2 by induction. To use the inductive assumptions easily we have to reformulate the conditions (1.35). The first two conditions (1.33), (1.34) have the property that they hold for $k-1$, if they hold for $k$. We will replace (1.35) by an equivalent condition which has this property also. By Proposition 2 of [3] the bounds $|\partial U-1|<\alpha_{0} \eta^{2}$ imply $\left|\partial \bar{U}^{j}-1\right|<\alpha_{0} L^{2 j} \eta^{2}+2 C_{1}\left(\alpha_{0} L^{2 j} \eta^{2}\right)^{2}<2 \alpha_{0} L^{2 j} \eta^{2}$, $j=0,1, \ldots, k$ for $\alpha_{0}$ small. Let us take $j=k-1$ and let us apply Lemma 1 to $V_{0}=\bar{U}_{0}^{k-1}, V^{\prime} V_{0}=\left(\overline{U^{\prime} U_{0}}\right)^{k-1}=\tilde{U}^{\prime k-1} \bar{U}_{0}^{k-1}$. Of course all the assumptions are satisfied and we obtain $\left|V^{\prime}-1\right|=\left|\tilde{U}^{\prime k-1}-1\right|<8 d^{2} \alpha_{0}+\alpha_{1}$. We apply again the Lemma 1 to $V_{0}=\bar{U}_{0}^{k-2}, V^{\prime} V_{0}=\widetilde{U}^{\prime k-2} \bar{U}_{0}^{k-2}$ and the constant $\alpha_{1}$ replaced by $8 d^{2} \alpha_{0}+\alpha_{1}$, and we obtain $\left|\tilde{U}^{\prime k-2}-1\right|<8 d^{2} \alpha_{0} L^{-2}+8 d^{2} \alpha_{0}+\alpha_{1}$. Continuing these arguments we get $\left|\widetilde{U}^{j}-1\right|<8 d^{2} \alpha_{0} L^{-2(k-j-1)}+\ldots+8 d^{2} \alpha_{0} L^{-2}+8 d^{2} \alpha_{0}+\alpha_{1}$ $<8 d^{2} \alpha_{0} \frac{1}{1-L^{-2}}+\alpha_{1}<11 d^{2} \alpha_{0}+\alpha_{1}$. Thus the conditions (1.33)-(1.35) imply

$$
\left|\left(\overline{U^{\prime} U_{0}}\right)^{j}-\bar{U}_{0}^{j}\right|=\left|\tilde{U}^{j}-1\right|<11 d^{2} \alpha_{0}+\alpha_{1} \text { on } \Omega_{j}^{(j)} .
$$

We will assume that the above bounds hold instead of (1.35). For simplicity let us write $\alpha_{1}$ instead of the right-hand side above, so we assume that

$$
\left|\left(\overline{U^{\prime} U_{0}}\right)^{j}-\bar{U}_{0}^{j}\right|=\left|\tilde{U}^{j}-1\right|<\alpha_{1} \quad \text { on } \quad \Omega_{j}^{(j)}, \quad j=0,1, \ldots, k .
$$

Now we are ready to give an inductive proof of the following 
Theorem 4. There exists a constant $c_{1}$ such that for arbitrary $U_{0}, U^{\prime} U_{0}$ satisfying (1.33), (1.34), (1.66) with $\alpha_{0}+\alpha_{1} \leqq c_{1}$ there exists exactly one gauge transformation $u$ satisfying (1.29) and such that the conditions (1.37), (1.38), (1.62) hold for the configuration $U_{1}=U^{u^{-1}}$.

Of course this theorem implies Theorem 2. Proposition 3 implies that it is enough to prove (1.37), (1.38) and

$$
|A|<B_{1}^{\prime}\left(\alpha_{0}+\alpha_{1}\right)\left(L^{j} \eta\right)^{-1} \quad \text { on } \quad \Omega_{j}, B_{1}^{\prime}=C_{1}^{\prime} 5 B_{0},
$$

with an absolute constant $C_{1}^{\prime}$, i.e. a constant depending on $d$ and $L$ only. In the rest of this paper we will be occupied with a proof of these conditions.

A proof of the first step, for $k=1$, will be included in a proof of the general step. Thus let us assume that Theorem 4 holds for some $k-1$ and we will prove it for $k$. Let us take configurations $U_{0}, U^{\prime} U_{0}$ satisfying the conditions (1.33), (1.34), (1.66). It was already noticed that they imply the same conditions for $k-1$ (we do not consider the set $\Omega_{k}$ ). Applying the inductive hypothesis we get a gauge transformation $u_{1}$ such that

$$
\begin{gathered}
\left(\overline{R_{0} u_{1}}\right)^{j}(y)=1 \quad \text { for } y \in \Lambda_{j}, \quad j=0,1, \ldots, k-2, \text { and } \\
\text { for } y \in \Lambda_{k-1} \cup B\left(\Lambda_{k}\right), \quad j=k-1, \\
U_{1}=U^{\prime u_{1}^{-1}}=e^{i L \eta A^{\prime}}=e^{i \eta A}, \quad|A|<B_{1}\left(\alpha_{0}+\alpha_{1}\right)\left(L^{j} \eta\right)^{-1}, \\
\left|\nabla_{U_{0}}^{\eta} A\right|<B_{1}\left(\alpha_{0}+\alpha_{1}\right)\left(L^{j} \eta\right)^{-2} \quad \text { on } \quad \Omega_{j}, \quad j=0,1, \ldots, k-1 .
\end{gathered}
$$

The other conditions in (1.37), (1.38), and (1.62) hold also, but we will not need them. It is clear from (1.69) that we have to improve the bounds on the domain $\Omega_{k}$.

Let us consider the gauge transformation $u_{1}$. From (64)-(87) of [3] it follows that $u_{1}$ is determined uniquely in terms of $U_{1}$ and is given by (106). From (105) and (106) we have for $x \in B^{j}\left(\Lambda_{j}\right)$,

$$
\begin{aligned}
\left|u_{1}(x)-1\right| & \leqq \sum_{n=j-1}^{0}\left(\left|\left(\overline{\bar{R}_{0, x_{n+1}}^{n} \overline{\bar{U}}_{1}^{n}}\right)-1\right|+\left|\left(\bar{R}_{0, x_{n+1}}^{n} \bar{U}_{1}^{n}\right)\left(\Gamma_{x_{n+1}, x_{n}}\right)-1\right|\right) \\
& \leqq 2 \sum_{n=0}^{j-1} \max _{x \in B\left(x_{n+1}\right)}\left|\frac{1}{i} \log \left(\bar{R}_{0, x_{n+1}}^{n} \bar{U}_{1}^{n}\right)\left(\Gamma_{x_{n+1}, x}\right)\right| .
\end{aligned}
$$

Using (130) and (1.69) we obtain

$$
\begin{aligned}
& \left|\frac{1}{i} \log \left(\bar{R}_{0, x_{n+1}}^{n} \bar{U}_{1}^{n}\right)\left(\Gamma_{x_{n+1}, x}\right)\right| \leqq\left|Q_{n}\left(U_{0}, \eta A\right)\right|\left(\Gamma_{x_{n+1}, x}\right) \\
& \quad+O\left(\left(\left|Q_{n}\left(U_{0}, \eta A\right)\right|\left(\Gamma_{x_{n+1}, x}\right)\right)^{2}\right)<2 B_{1}\left(\alpha_{0}+\alpha_{1}\right) L^{n-j} d L \\
& \quad+O\left(\left(2 B_{1}\left(\alpha_{0}+\alpha_{1}\right) L^{n-j} d L\right)^{2}\right) \leqq 4 d B_{1}\left(\alpha_{0}+\alpha_{1}\right) L^{n+l-j}
\end{aligned}
$$

for $\alpha_{0}+\alpha_{1}$ sufficiently small, hence

$$
\left|u_{1}(x)-1\right|<8 d B_{1}\left(\alpha_{0}+\alpha_{1}\right) \frac{1}{1-L^{-1}} \leqq 16 d B_{1}\left(\alpha_{0}+\alpha_{1}\right), \quad x \in \Omega_{0} .
$$

The same argument can be applied to the averages $\overline{R_{0} u_{1}^{n}}$, and we get

$$
\left|\left({\overline{R_{0}}}_{0}^{n}\right)\left(x_{n}\right)-1\right|<16 d B_{1}\left(\alpha_{0}+\alpha_{1}\right), \quad x_{n} \in \Omega_{n}^{(n)} .
$$


From (108) and (1.71) we obtain also

$$
\left|\left({\overline{R_{0}}}_{1}^{n}\right)^{-1}\left(x_{n+1}\right)\left(\bar{R}_{0, x_{n+1}}^{n} \overline{R_{0} u_{0}^{n}}\right)\left(x_{n}\right)-1\right|<4 d B_{1}\left(\alpha_{0}+\alpha_{1}\right) L^{n+1-j}
$$

for $x_{n} \in B\left(x_{n+1}\right), \quad x_{n+1} \in B^{j-n-1}\left(\Lambda_{j}\right), n=0, \ldots, j-1, j=1, \ldots, k-1$ (we take $\Lambda_{k-1} \cup B\left(\Lambda_{k}\right)$ as $\left.\Lambda_{k-1}\right)$.

We will use only the properties (1.69), (1.73), (1.74) of the configurations $u_{1}, U_{1}$ in the future. They are satisfied in the case $k=1$ also, if we take $u_{1}=1, U^{\prime}=U_{1}$, because then by Lemma 1 we have $\left|U_{1}-1\right|<4 d^{2} \alpha_{0}+\alpha_{1}$ and $U_{1}=e^{i A^{\prime}}=e^{i L^{-1} A}$ with $A$ satisfying the bounds (1.69), at least for $B_{1}$ not too small, e.g. for $B_{0}$ satisfying $5 d L B_{0} \geqq 8 d^{2}$. The conditions (1.73), (1.74) are important because we have analyzed the averaging operations for gauge transformations in [3] assuming that they satisfy these conditions. We will use the results of that paper.

The gauge transformation $u_{1}$ transforms the configuration $U^{\prime}$ into the configuration $U_{1}$ which is sufficiently regular and close to 1 . We want to find another gauge transformation $u^{\prime}$ which transforms $U_{1}$ into a configuration $U_{1}^{u^{\prime}-1}$ satisfying the conditions (1.37), (1.38), (1.67) and which satisfies the conditions

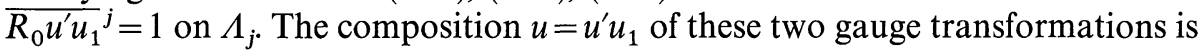
the transformation we are looking for. The conditions which have to be satisfied for $u^{\prime}$ lead to restrictions on $u^{\prime}$, e.g. $u^{\prime}$ has to be a small configuration also, and they lead to equations on $u^{\prime}$. We will analyze these equations and we will prove that under proper conditions they have a unique solution.

\section{Equations for the Gauge Transformation $\boldsymbol{u}^{\prime}$}

We fix a configuration $U_{0}$ and we omit it in formulas below, for example we write $D$ instead of $D_{U_{0}}^{\eta}$. We are looking for a configuration $u^{\prime}$ such that $U_{1}^{u^{\prime}-1}$ satisfies the conditions (1.37), (1.38), (1.62). In particular (1.62) and (1.69) imply

$$
\begin{gathered}
\left|u^{\prime-1}\left(b_{-}\right) R_{0, b} u^{\prime}\left(b_{+}\right)-1\right| \leqq\left|u^{\prime-1}\left(b_{-}\right) U_{1, b} R_{0, b} u^{\prime}\left(b_{+}\right)-1\right| \\
+\left|u^{\prime-1}\left(b_{-}\right)\left(U_{1, b}-1\right) R_{0, b} u^{\prime}\left(b_{+}\right)\right|<2 B_{1}\left(\alpha_{0}+\alpha_{1}\right) \eta\left(L^{j} \eta\right)^{-1} \\
\text { on } \Omega_{j}, \quad j=1, \ldots, k-1 .
\end{gathered}
$$

Reasoning as in $(1.70)-(1.72)$ we have $\left|u^{\prime} u_{1}-1\right|<16 d B_{1}\left(\alpha_{0}+\alpha_{1}\right)$. From the inequality (1.72) for $u_{1}$ we get

$$
\left|u^{\prime}-1\right|<32 d B_{1}\left(\alpha_{0}+\alpha_{1}\right) .
$$

The estimates (1.75), (1.76) imply that $u^{\prime}$ satisfies the regularity conditions (176), (177) [3] on $\Omega_{j} \subset T_{L^{-j}}$ with $\alpha_{4}=32 d B_{1}\left(\alpha_{0}+\alpha_{1}\right)$. The estimates (1.73), (1.74) together with the condition $\overline{R_{0} u_{1}^{j}}=1$ on $\Lambda_{j}$ imply that $u_{1}$ satisfies (166), (167) [3] on $B^{j}\left(\Lambda_{j}\right)$ $C T_{L^{-j}}$ with $\alpha_{3}=16 d B_{1}\left(\alpha_{0}+\alpha_{1}\right)$, thus for $\alpha_{0}+\alpha_{1}$ sufficiently small the assumptions of Proposition 10 are satisfied and we have the representation (213) and the bounds (214) [3].

We have to construct the configuration $u^{\prime}$, so we have to change somewhat our point of view. The above estimates show that it is natural to assume the regularity conditions (176), (177) on $\Omega_{j} \subset T_{L^{-j}}$, with some constant $\alpha_{4}$ to be determined later. Here it is more convenient to work with regularity conditions of the type (207) [3]. 
Thus we consider the set of configurations $u^{\prime}=e^{i \lambda}$ with $\lambda$ satisfying

$$
|\lambda|<\alpha_{4},|(D \lambda)(b)|<\alpha_{4}\left(L^{j} \eta\right)^{-1} \quad \text { for } \quad b \in \Omega_{j}, \quad j=0,1, \ldots, k-1,
$$

where $\alpha_{4}$ is sufficiently small, but otherwise arbitrary (i.e., not necessarily given by $\left.\alpha_{4}=32 d B_{1}\left(\alpha_{0}+\alpha_{1}\right)\right)$. These conditions imply the regularity conditions (176), (177) on $\Omega_{j} \subset T_{L^{-j}}$, with $4 \alpha_{4}$ instead of $\alpha_{4}$.

The configuration $u_{1}$ is fixed and determined by $U_{1}$, hence we keep the same meaning of $\alpha_{3}$ as before (i.e. $\alpha_{3}=16 d B_{1}\left(\alpha_{0}+\alpha_{1}\right)$ ). The transformation $u^{\prime} u_{1}$ has to satisfy the conditions (1.29), and $u_{1}$ satisfies (1.68). Of course $\left({\overline{R_{0} u_{1}}}^{k-1}\right)(x)=1$ for $x \in B\left(\Lambda_{k}\right)$ implies $\left({\overline{R_{0} u_{1}}}^{k}\right)(y)=1$ for $y \in \Lambda_{k}$, hence $u_{1}$ satisfies the conditions (1.29) and we may write these conditions for $u^{\prime} u_{1}$ in the following way

$$
\tilde{u}^{\prime j}={\overline{R_{0} u^{\prime} u_{1}}}^{j}\left({\overline{R_{0} u_{1}}}^{j}\right)^{-1}=1 \quad \text { on } \quad \Lambda_{j}, \quad j=0,1, \ldots, k,
$$

or equivalently as

$$
Q^{\prime}\left(u_{1}, \lambda\right)=0 \quad \text { on } \quad \mathfrak{B}_{k}, \quad \lambda=\frac{1}{i} \log u^{\prime}
$$

where

$$
Q^{\prime}\left(u_{1}, \lambda, y\right)=Q_{j}^{\prime}\left(u_{1}, \lambda, y\right) \text { for } y \in \Lambda_{j} \subset \mathfrak{B}_{k} .
$$

We get a second equation for $\lambda$ using (1.38):

$$
R D^{*} \frac{1}{i \eta} \log U_{1}^{u^{\prime-1}}=0 \text {. }
$$

We have

$$
\begin{aligned}
\left(U_{1}^{u^{\prime}-1}\right)_{b} & =u^{\prime-1}\left(b_{-}\right) U_{1, b} R_{0, b} u^{\prime}\left(b_{+}\right)=e^{i \eta R\left(u^{\prime}-1\left(b_{-}\right)\right) A_{b}} e^{-i \lambda\left(b_{-}\right)} e^{i R_{0, b} \lambda\left(b_{+}\right)} \\
& =e^{i \eta R\left(u^{\prime}-1\left(b_{-}\right)\right) A_{b}} e^{-i \lambda\left(b_{-}\right)} e^{i \lambda\left(b_{-}\right)+i \eta(D \lambda)(b)}
\end{aligned}
$$

and using the formulas (32), (36)-(41) [3] we obtain

$$
\left(U_{1}^{u^{\prime}-1}\right)_{b}=e^{i \eta R\left(u^{\prime}-1\left(b_{-}\right)\right) A_{b}} e^{i \eta g\left(i \mathrm{ad}_{\lambda\left(b_{-}\right)}\right)(D \lambda)(b)+i \eta^{2} \widetilde{\mho}_{1}\left(\lambda\left(b_{-}\right),(D \lambda)(b)\right)},
$$

where the Lie algebra valued function $\mathfrak{F}_{1}$ satisfies the bound

$$
\left|\mathfrak{F}_{1}\left(\lambda\left(b_{-}\right),(D \lambda)(b)\right)\right| \leqq O(1)|(D \lambda)(b)|^{2} .
$$

We assume of course that $\alpha_{4}$ is sufficiently small. Using (28) and (31) [3], we get

$$
\frac{1}{i \eta} \log \left(U_{1}^{u^{\prime-1}}\right)_{b}=R\left(u^{\prime-1}\left(b_{-}\right)\right) A_{b}+g\left(\operatorname{iad}_{\lambda(b-)}\right)(D \lambda)(b)+\eta \mathfrak{F}_{2}\left(\lambda\left(b_{-}\right),(D \lambda)(b), A_{b}\right),
$$

where

$$
\left|\mathfrak{F}_{2}\left(\lambda\left(b_{-}\right),(D \lambda)(b), A_{b}\right)\right| \leqq O(1)\left|A_{b}\right||(D \lambda)(b)|
$$

Now we apply the derivative $D^{*}$ to the expression on the right-hand side of (1.84). $\eta D^{*}$ is a bounded operator, hence $D^{*} \eta \mathfrak{F}_{1}$ satisfies the bound (1.85). The operators $R\left(u^{\prime-1}\left(b_{-}\right)\right)=e^{-i \operatorname{ad}_{\lambda\left(b_{-}\right)}}$and $g\left(i \operatorname{ad}_{\lambda\left(b_{-}\right)}\right)$are given by power series in $\operatorname{ad}_{\lambda_{\left(b_{-}\right)}}$. Let us 
consider for example a term $\left(\operatorname{ad}_{\lambda\left(b_{-}\right)}\right)^{n} A_{b}$,

$$
\begin{aligned}
\eta D_{\mu}^{*}\left(\operatorname{ad}_{\lambda(x)}\right)^{n} A_{\mu}(x) & =R\left(U_{0}\left(x, x-\eta e_{\mu}\right)\right)\left(\operatorname{ad}_{\lambda\left(x-\eta e_{\mu}\right)}\right)^{n} A_{\mu}\left(x-\eta e_{\mu}\right)-\left(\operatorname{ad}_{\lambda(x)}\right)^{n} A_{\mu}(x) \\
& =\left(\operatorname{ad}_{R\left(U_{0}\left(x, x-\eta e_{\mu}\right)\right) \lambda\left(x-\eta e_{\mu}\right)}\right)^{n} R\left(U_{0}\left(x, x-\eta e_{\mu}\right)\right) A_{\mu}\left(x-\eta e_{\mu}\right) \\
& -\left(\operatorname{ad}_{\lambda(x)}\right)^{n} A_{\mu}(x) \\
& =\left(\eta \operatorname{ad}_{\left(D_{\mu}^{*} \lambda\right)(x)}+\operatorname{ad}_{\lambda(x)}\right)^{n}\left(\eta\left(D_{\mu}^{*} A_{\mu}\right)(x)+A_{\mu}(x)\right) \\
& -\left(\operatorname{ad}_{\lambda(x)}\right)^{n} A_{\mu}(x),
\end{aligned}
$$

hence

$$
D_{\mu}^{*}\left(\operatorname{ad}_{\lambda(x)}\right)^{n} A_{\mu}(x)=\left(\operatorname{ad}_{\lambda(x)}\right)^{n}\left(D_{\mu}^{*} A_{\mu}\right)(x)+P_{n}\left(\lambda(x), D_{\mu}^{*} \lambda(x), A_{\mu}(x), A_{\mu}\left(x-\eta e_{\mu}\right)\right),
$$

and

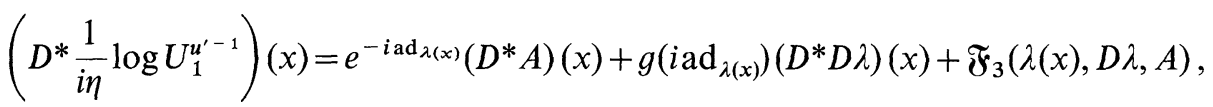

where $\mathfrak{F}_{3}$ depends on $(D \lambda)(b), A_{b}$ for $b \in \operatorname{st}(x)$ and satisfies

$$
\left|\mathfrak{F}_{3}(\lambda(x), D \lambda, A)\right| \leqq O(1)|D \lambda||A| \text {. }
$$

Equations (1.79), (1.80) can be written as

$$
\begin{gathered}
R g\left(\operatorname{iad}_{\lambda}\right) D^{*} D \lambda+\operatorname{Re}^{-i \mathrm{ad}_{\lambda}} D^{*} A+R \mathfrak{F}_{3}(\lambda, D \lambda, A)=0, \\
Q^{\prime}\left(u_{1}, \lambda\right)=0 .
\end{gathered}
$$

The function $Q^{\prime}\left(u_{1}, \lambda\right)$ is almost equal to $Q^{\prime} \lambda$, the linear averaging operator used in the definition of the operator $R$, the error is of second order in $\alpha_{3}, \alpha_{4}$. If it was equal to $Q^{\prime} \lambda$, then $D^{*} D \lambda=\Delta \lambda$ would belong to the subspace $R=\Delta N\left(Q^{\prime}\right)$ and we would have

$$
R g\left(i \operatorname{ad}_{\lambda}\right) \Delta \lambda=R \Delta \lambda+O\left(\alpha_{4}\right) \Delta \lambda=\left(\Delta+Q^{*} a Q^{\prime}\right) \lambda+O\left(\alpha_{4}\right) \Delta \lambda
$$

Thus the main linear part in $\lambda$ of the left-hand side of (1.90) would be given by the invertible operator, the remaining terms being small. We will reduce the problem (1.90) to such a situation by a change of variables. We want to find a transformation of variables $\lambda$ which linearizes the function $Q^{\prime}\left(u_{1}, \lambda\right)$, more exactly transforms it into the function $Q^{\prime} \lambda$. This transformation has to be applied to Eqs. (1.90) also, hence it has to be sufficiently regular. Of course such a problem is highly indeterminate because a number of variables involved is much bigger than a number of conditions. In the next section we will find one solution of this problem. Let us introduce the operators

$$
H^{\prime}=G^{\prime 2} Q^{*}\left(Q^{\prime} G^{\prime 2} Q^{* *}\right)^{-1}, \quad G^{\prime}=\left(\Delta+Q^{*} a Q^{\prime}\right)^{-1} .
$$

They were investigated in [4], and the following inequality can be obtained from the results of this paper:

$$
\left|\left(H^{\prime} X\right)(x)\right|,\left|\left(\nabla H^{\prime} X\right)(x)\right| \leqq B_{0}^{\prime}\left[1,\left(L^{j} \eta\right)^{-1}\right]|X| \text { for } \quad x \in \Omega_{j},
$$


where

$$
\left(H^{\prime} X\right)(x)=\sum_{y^{\prime} \in \mathfrak{B}_{k}}\left(L^{j \prime} \eta\right)^{d} H^{\prime}\left(x, y^{\prime}\right) X\left(y^{\prime}\right),
$$

and $B_{0}^{\prime}$ is an absolute constant (depending on $d$ and $L$ only).

In the next section we will prove that for $\alpha_{3}, \alpha_{4}$ sufficiently small there exists a function $D^{\prime}\left(u_{1}, \lambda\right)$ such that the change of variables $\lambda \rightarrow \lambda-H^{\prime} D^{\prime}\left(u_{1}, \lambda\right)$ transforms the function $Q^{\prime}\left(u_{1}, \lambda\right)$ into $Q^{\prime} \lambda$. The function $D^{\prime}\left(u_{1}, \lambda\right)$ is analytic in $\lambda$, defined on a set of $\lambda$ satisfying (1.77) with $1 / 2 \alpha_{4}$ instead of $\alpha_{4}$, and is bounded by $C_{2}^{\prime}\left(\alpha_{3}+\alpha_{4}\right) \alpha_{4}$, and also by $\frac{1}{2 B_{0}^{\prime}} \alpha_{4}$. This change of variables applied to Eqs. (1.90) gives the following equations

$$
\begin{gathered}
R g\left(\operatorname{iad}_{\lambda-H^{\prime} D^{\prime}\left(u_{1}, \lambda\right)}\right) \Delta\left(\lambda-H^{\prime} D^{\prime}\left(u_{1}, \lambda\right)\right)-R \exp \left(-i \operatorname{ad}_{\lambda-H^{\prime} D^{\prime}\left(u_{1}, \lambda\right)}\right) D^{*} A \\
-R \mathfrak{F}_{3}\left(\lambda-H^{\prime} D^{\prime}\left(u_{1}, \lambda\right), D \lambda-D H^{\prime} D^{\prime}\left(u_{1}, \lambda\right), A\right)=0, \\
Q^{\prime} \lambda=0 .
\end{gathered}
$$

The first term in the first equation can be written as

$$
\left[I+R\left[g\left(i \operatorname{ad}_{\lambda-H^{\prime} D^{\prime}\left(u_{1}, \lambda\right)}\right)-1\right] R\right] \Delta \lambda,
$$

because $R \Delta \lambda=\Delta \lambda$ for $\lambda$ satisfying $Q^{\prime} \lambda=0$. For $\alpha_{4}$ small the second operator above has a small norm. Let us recall that from Theorems 3.1, 3.2 of [4] it follows that $|R f| \leqq B_{0}^{\prime}|f|$, hence the operator $R[\ldots] R$ has a $L^{\infty}$ norm bounded by $O\left(\alpha_{4}\right) B_{0}^{\prime 2}$. We assume that $\alpha_{4}$ is so small that $O\left(\alpha_{4}\right) B_{0}^{\prime 2} \leqq \frac{1}{2}$. Then the operator in the square bracket in (1.94) is invertible. Multiplying Eq. (1.93) by an inverse operator we obtain the equations

$$
\Delta \lambda-R D^{*} A-R \mathfrak{F}_{4}\left(\lambda, D \lambda, A, D^{*} A\right)=0, \quad Q^{\prime} \lambda=0 .
$$

Now we will investigate properties of the function $R \mathfrak{F}_{4}$. Let us denote by $V$ the operator $g(\ldots)-1$ in the internal bracket $[\ldots]$ in (1.94). It is a very simple local operator of the form $(V f)(x)=V(x) f(x)$, where $V(x)$ is a linear operator on the Lie algebra $\mathfrak{g}$, satisfying the bound $|V| \leqq O\left(\alpha_{4}\right)$. The operator in (1.94) acting on $\Delta \lambda$ is equal to $I+R V R$ and its inverse is given by the Neumann series

$$
(I+R V R)^{-1}=I+\sum_{n=1}^{\infty}(-1)^{n}(R V R)^{n}=I+\sum_{n=1}^{\infty}(-1)^{n} R(V R)^{n} .
$$

Let us consider the term with the function $\mathfrak{F}_{3}$ in Eq. (1.93). Using the inequality (1.89) we can bound a value of this function at a point $x \in \Omega_{j}$ by

$$
\begin{aligned}
& O(1) \max _{b \in s t(x)}\left|(D \lambda)(b)-\left(D H^{\prime} D^{\prime}\left(u_{1}, \lambda\right)\right)(b)\right|\left|A_{b}\right|<O(1) \alpha_{4}\left(L^{j} \eta\right)^{-1} B_{1}\left(\alpha_{0}+\alpha_{1}\right)\left(L^{j} \eta\right)^{-1} \\
& \quad=O(1) B_{1}\left(\alpha_{0}+\alpha_{1}\right) \alpha_{4}\left(L^{j} \eta\right)^{-2} .
\end{aligned}
$$

Thus it is a function with a bounded norm $|\cdot|_{(-2)}$.

The operators $R$ and $V$ are bounded in this norm, and we have

$$
|R f|_{(-2)} \leqq B_{0}^{\prime}|f|_{(-2)}, \quad|V f|_{(-2)} \leqq O\left(\alpha_{4}\right)|f|_{(-2)} .
$$


This and (1.96) implies that the operator $(I+R V R)^{-1}$ applied to the last term in (1.93) gives a term bounded by $O(1) B_{1}\left(\alpha_{0}+\alpha_{1}\right) \alpha_{4}\left(L^{j} \eta\right)^{-2}$ on $\Omega_{j}$. We get the same bound for the term with $D^{*} A$ (but with $R D^{*} A$ subtracted), and with $\Delta H^{\prime} D^{\prime}\left(u_{1}, \lambda\right)$. Thus we have the bound

$$
\left|R F_{4}\left(\lambda, D \lambda, A, D^{*} A\right)\right| \leqq C_{4}^{\prime} B_{1}\left(\alpha_{0}+\alpha_{1}\right) \alpha_{4}\left(L^{j} \eta\right)^{-2} \text { on } \Omega_{j}
$$

for $j<k$.

Equations (1.95) can be changed into the equivalent equation

$$
\lambda=G^{\prime} R D^{*} A+G^{\prime} R \mathfrak{F}_{4}\left(\lambda, D \lambda, A, D^{*} A\right) .
$$

If $\lambda$ is a solution of this equation, then $\lambda$ satisfies both Eqs. (1.95) because $Q^{\prime} G^{\prime} R=Q^{\prime} G^{\prime}\left(I-G^{\prime} Q^{* *}\left(Q^{\prime} G^{\prime 2} Q^{* *}\right)^{-1} Q^{\prime} G^{\prime}\right)=0$. Of course (1.95) imply (1.100). A solution of this equation may be interpreted as a fixed point of the transformation given by the right-hand side of the equation. Let us denote it by $\mathfrak{F}(\lambda)$. We will prove that for $\alpha_{0}+\alpha_{1}$ sufficiently small and for $\alpha_{4}$ chosen properly there exists exactly one fixed point of $\mathfrak{F}$.

At first we will find domains in $\lambda$ which are invariant under the transformation $\mathfrak{F}$. One of the results of [4], Theorem 3.1, tells us that $G^{\prime}$ is a bounded operator from a space with the norm $|\cdot|_{(-2)}$ into a space with the norm $|\cdot|$ for functions, and the norm $|\cdot|_{(-1)}$ for their first derivatives. Thus we have

$$
\begin{aligned}
|\mathfrak{F}(\lambda)| & \leqq B_{0}^{\prime} B_{1}\left(\alpha_{0}+\alpha_{1}\right)+B_{0}^{\prime} C_{4}^{\prime} B_{1}\left(\alpha_{0}+\alpha_{1}\right) \alpha_{4}, \\
|D \mathfrak{F}(\lambda)| & \leqq\left(B_{0}^{\prime} B_{1}\left(\alpha_{0}+\alpha_{1}\right)+B_{0}^{\prime} C_{4}^{\prime} B_{1}\left(\alpha_{0}+\alpha_{1}\right) \alpha_{4}\right)\left(L^{j} \eta\right)^{-1} \text { on } \Omega_{j},
\end{aligned}
$$

if $\lambda$ belongs to the space

$$
\left\{\lambda:|\lambda|<\beta \alpha_{4},|D \lambda|<\beta \alpha_{4}\left(L^{j} \eta\right)^{-1} \quad \text { on } \quad \Omega_{j}, \quad j=0,1, \ldots, k-1\right\}, \quad \beta \leqq \frac{1}{2},
$$

or simply

$$
\left\{\lambda:|\lambda|,|D \lambda|_{(-1)}<\beta \alpha_{4}\right\} \text {. }
$$

The function $\mathfrak{F}$ is obviously an analytic function in $\lambda$ and we consider configurations $\lambda$ with values in the complexified algebra. The function $\mathbb{F}$ maps the domain (1.102) into itself if $C_{4}^{\prime} \alpha_{4} \leqq 1$ and

$$
B_{0}^{\prime}\left(1+C_{4}^{\prime} \alpha_{4}\right) B_{1}\left(\alpha_{0}+\alpha_{1}\right) \leqq 2 B_{0}^{\prime} B_{1}\left(\alpha_{0}+\alpha_{1}\right) \leqq \beta \alpha_{4},
$$

hence if

$$
\alpha_{4} \leqq \frac{1}{C_{4}^{\prime}}, \alpha_{0}+\alpha_{1} \leqq \frac{1}{2 B_{0}^{\prime} B_{1}} \beta \alpha_{4}
$$

Now we will prove that for $\alpha_{4}$ sufficiently small the transformation is contractive. We have

$$
\begin{aligned}
\mathfrak{F}\left(\lambda_{1}\right)-\mathfrak{F}\left(\lambda_{2}\right)= & G^{\prime}\left(R \mathfrak{F}_{4}\left(\lambda_{1}, D \lambda_{1}, A, D^{*} A\right)-R \mathfrak{F}_{4}\left(\lambda_{2}, D \lambda_{2}, A, D^{*} A\right)\right) \\
= & G^{\prime} \int_{0}^{1} d t \frac{d}{d t} R \mathfrak{F}_{4}\left(t \lambda_{1}+(1-t) \lambda_{2}, t D \lambda_{1}+(1-t) D \lambda_{2}, A, D^{*} A\right) \\
= & G^{\prime} \int_{0}^{1} d t \frac{d}{d \tau} R \mathfrak{F}_{4}\left(t \lambda_{1}+(1-t) \lambda_{2}+\tau\left(\lambda_{1}-\lambda_{2}\right), t D \lambda_{1}\right. \\
& \left.+(1-t) D \lambda_{2}+\tau\left(D \lambda_{1}-D \lambda_{2}\right), A, D^{*} A\right)\left.\right|_{\tau=0} .
\end{aligned}
$$


Let us take $\lambda_{1}, \lambda_{2}$ from the domain (1.102) with $\beta=\frac{1}{4}$ and let us apply the Cauchy formula for the derivative of the analytic function in $\tau$ above. We have

$$
\begin{aligned}
\mathfrak{F}\left(\lambda_{1}\right)-\mathfrak{F}\left(\lambda_{2}\right)= & G^{\prime} \int_{0}^{1} d t \frac{1}{2 \pi i} \int_{|\tau|=r} \frac{d \tau}{\tau^{2}} R \mathfrak{F}_{4}\left(t \lambda_{1}+(1-t) \lambda_{2}+\tau\left(\lambda_{1}-\lambda_{2}\right), t D \lambda_{1}\right. \\
& \left.+(1-t) D \lambda_{2}+\tau\left(D \lambda_{1}-D \lambda_{2}\right), A, D^{*} A\right)
\end{aligned}
$$

with $r=\left(4 \max \left\{\left|\lambda_{1}-\lambda_{2}\right|,\left|D \lambda_{1}-D \lambda_{2}\right|_{(-1)}\right\}\right)^{-1} \alpha_{4}$. The argument $t \lambda_{1}+(1-t) \lambda_{2}$ $+\tau\left(\lambda_{1}-\lambda_{2}\right)$ belongs to the domain (1.102) with $\beta=\frac{1}{2}$, and we use the inequality (1.99). We get

$$
\begin{aligned}
& \left|\mathfrak{F}\left(\lambda_{1}\right)-\mathfrak{F}\left(\lambda_{2}\right)\right|,\left|D\left(\mathfrak{F}\left(\lambda_{1}\right)-\mathfrak{F}\left(\lambda_{2}\right)\right)\right|_{(-1)} \\
& \quad \leqq B_{0}^{\prime} C_{4}^{\prime} B_{1}\left(\alpha_{0}+\alpha_{1}\right) 4 \max \left\{\left|\lambda_{1}-\lambda_{2}\right|,\left|D \lambda_{1}-D \lambda_{2}\right|_{(-1)}\right\},
\end{aligned}
$$

and by (1.103)

$$
4 C_{4}^{\prime} B_{0}^{\prime} B_{1}\left(\alpha_{0}+\alpha_{1}\right) \leqq 2 C_{4}^{\prime} \beta \alpha_{4}=\frac{1}{2} C_{4}^{\prime} \alpha_{4} \leqq \frac{1}{2} \text { for } \beta=\frac{1}{4} .
$$

Thus the transformation $\mathfrak{F}$ maps the domain (1.102) with $\beta=\frac{1}{4}$ into itself and is contractive if the inequality (1.103) holds with $\beta=\frac{1}{4}$. The contraction mapping theorem implies that there exists a unique fixed point of this transformation in the considered domain. To get a best uniqueness result we have to take a largest possible $\alpha_{4}$. It is independent of $\alpha_{0}+\alpha_{1}$. To get best bounds on the solution we have to take a smallest possible $\alpha_{4}$, hence $\alpha_{4}=8 B_{0}^{\prime} B_{1}\left(\alpha_{0}+\alpha_{1}\right)$, and then we have to assume that $\alpha_{0}+\alpha_{1}$ is so small that all the conditions on $\alpha_{0}+\alpha_{1}, \alpha_{4}$ are satisfied.

The solution $\lambda$ of Eq. (1.100) determines the configuration $u^{\prime}=\exp \left[i\left(\lambda-H^{\prime} D^{\prime}\left(u_{1}, \lambda\right)\right)\right]$ we are looking for. Let us formulate the obtained results in the following

Proposition 5. There exist positive constants $c_{2}, c_{3}$, depending on $d$ and L only, such that for an arbitrary configuration $U_{1}$ satisfying $(1.69)$, and for the configuration $u_{1}$ determined by $U_{1}$ and satisfying (1.68), (1.73), (1.74), if $\alpha_{0}+\alpha_{1} \leqq c_{2}$, then there exists a configuration $u^{\prime}=e^{i \lambda}$ satisfying the equations

$$
R D^{*} \frac{1}{i \eta} \log U_{1}^{u^{\prime}-1}=0, \overline{R_{0} u^{\prime} u_{1}^{j}}=1 \quad \text { on } \quad \Lambda_{j}, j=0,1, \ldots, k
$$

and the bounds

$$
|\lambda|,|D \lambda|_{(-1)}<8 B_{0}^{\prime} B_{1}\left(\alpha_{0}+\alpha_{1}\right) .
$$

Such a configuration $u^{\prime}$ is unique in the domain

$$
|\lambda|,|D \lambda|_{(-1)}<c_{3} .
$$

The part of this proposition concerning the existence of $u^{\prime}$ was proved completely. The uniqueness follows from the fact that the image of a set $\{\lambda:|\lambda|$, $\left.|D \lambda|_{(-1)}<\frac{1}{2} \alpha_{4}\right\}$ by the transformation $\lambda \rightarrow \lambda-H^{\prime} D^{\prime}\left(u_{1}, \lambda\right)$ contains the set $\left\{\lambda^{\prime}:\left|\lambda^{\prime}\right|\right.$, $\left|D \lambda^{\prime}\right|_{(-1)}<\frac{1}{4} \alpha_{4}$ for $\alpha_{4}$ sufficiently small. This follows from results of the next section.

Now let us consider implications of the proposition concerning the inductive proof of the Theorem 4. It gives us a gauge transformation $u=u^{\prime} u_{1}$ such that the conditions (1.29), (1.37), (1.38) are satisfied. This follows from the inductive 
construction of $U_{1}$ and from (1.107). The bounds (1.108) and the representation (1.84) imply

$$
\begin{aligned}
\left|\frac{1}{i \eta} \log \left(U_{1}^{u^{\prime}-1}\right)_{b}\right| \leqq & \left|A_{b}\right|+\left|g\left(i \operatorname{ad}_{\lambda\left(b_{-}\right)}\right)(D \lambda)(b)\right|+O(1) \eta|(D \lambda)(b)|\left|A_{b}\right| \\
\leqq & 2\left|A_{b}\right|+2|(D \lambda)(b)|<2 B_{1}\left(\alpha_{0}+\alpha_{1}\right)\left(L^{j} \eta\right)^{-1} \\
& +16 B_{0}^{\prime} B_{1}\left(\alpha_{0}+\alpha_{1}\right)\left(L^{j} \eta\right)^{-1} \\
= & \left(2+16 B_{0}^{\prime}\right) B_{1}\left(\alpha_{0}+\alpha_{1}\right)\left(L^{j} \eta\right)^{-1} \text { on } \Omega_{j}, j=0,1, \ldots, k-1,
\end{aligned}
$$

hence

$$
\begin{aligned}
\left|\frac{1}{i \eta} \log \left(U_{1}^{u^{\prime}-1}\right)_{b}\right| & <\left(2+16 B_{0}^{\prime}\right) L B_{1}\left(\alpha_{0}+\alpha_{1}\right)\left(L^{j} \eta\right)^{-1} \\
& =C_{1}^{\prime} B_{1}\left(\alpha_{0}+\alpha_{1}\right)\left(L^{j} \eta\right)^{-1} \text { on } \Omega_{j}, j=0,1, \ldots, k,
\end{aligned}
$$

where $C_{1}^{\prime}$ is an absolute constant depending on $d$ and $L$ only. Thus all the assumptions of Proposition 3 are satisfied and for $\alpha_{0}+\alpha_{1}$ sufficiently small it implies Theorem 4, except the uniqueness statement.

To prove the uniqueness let us assume that there are two transformations $u_{1}, u_{2}$ satisfying the conditions of the Theorem 4 . Then we have two configurations $U_{1}=U^{u_{1}^{-1}}, U_{2}=U^{u_{2}^{-1}}, U_{1}=e^{i \eta A_{1}}, U_{2}=e^{i \eta A_{2}}$. They satisfy all the conditions of the theorem, hence $A_{2}$ satisfies (1.37), (1.38), and both configurations $A_{1}, A_{2}$ satisfy the bounds (1.62). This implies that $u^{\prime}=u_{2} u_{1}^{-1}$ satisfies the regularity conditions (1.73), (1.74) with $k$ instead of $k-1$ and with a worse constant. This follows from Proposition 8 of [3]. We have

$$
u^{\prime} u_{1}=u_{2}, \text { hence }{\overline{R_{0} u^{\prime} u_{1}}}^{j}=1 \quad \text { on } \Lambda_{j}, \quad j=0,1, \ldots, k, \quad U_{1}^{u^{\prime-1}}=U_{2},
$$

thus $u^{\prime}$ is a solution of the problem described in Proposition 5. For $\alpha_{0}+\alpha_{1}$ sufficiently small the assumptions of this proposition are satisfied and we have the uniqueness property. In the considered case a configuration identically equal to 1 is a solution also, because $U_{1}$ satisfies the same conditions as $U_{2}$. The uniqueness implies $u^{\prime}=1$, or $u_{1}=u_{2}$. Thus we have completed the proof of Theorem 4 , and Theorem 2.

\section{E. A Construction of the Linearizing Transformation}

Let us consider the function $Q^{\prime}\left(u_{1}, \lambda^{\prime}\right)$. The configuration $u_{1}$ is fixed and we omit $u_{1}$ in the notations below. We want to construct a function $D^{\prime}(\lambda)$ for $\alpha_{3}, \alpha_{4}$ sufficiently small, whose values are configurations $X: \mathfrak{B}_{k} \rightarrow \mathfrak{g}$, such that the transformation

$$
\lambda^{\prime}=\lambda-H^{\prime} D(\lambda)
$$

changes the function $Q^{\prime}\left(\lambda^{\prime}\right)$ into the linear function $Q^{\prime} \lambda$ :

$$
Q^{\prime}\left(\lambda^{\prime}\right)=Q^{\prime}\left(\lambda-H^{\prime} D^{\prime}(\lambda)\right)=Q^{\prime} \lambda .
$$


Using the equality (213) [3] the above equation can be written in the following form:

$$
Q^{\prime} \lambda-Q^{\prime} H^{\prime} D^{\prime}(\lambda)+C^{\prime}\left(\lambda-H^{\prime} D^{\prime}(\lambda)\right)=Q^{\prime} \lambda
$$

hence

$$
-D^{\prime}(\lambda)+C^{\prime}\left(\lambda-H^{\prime} D^{\prime}(\lambda)\right)=0 .
$$

The function $D^{\prime}(\lambda)$ is a solution of the equation

$$
C^{\prime}\left(\lambda-H^{\prime} X\right)=X
$$

or a fixed point of the transformation

$$
X \rightarrow C^{\prime}\left(\lambda-H^{\prime} X\right) \text {. }
$$

The configurations $u_{1}, \lambda$ are treated as parameters and a solution of Eq. (1.117) depends on them.

At first we will find a set of functions $X$ which is transformed into itself by the transformation (1.118). Let us assume that

$$
|\lambda|<\frac{1}{2} \alpha_{4},|D \lambda|<\frac{1}{2} \alpha_{4}\left(L^{j} \eta\right)^{-1} \quad \text { on } \quad \Omega_{j}, \quad|X|<\frac{1}{2 B_{0}^{\prime}} \alpha_{4} .
$$

This implies

$$
\left|\lambda-H^{\prime} X\right|<\alpha_{4},\left|D\left(\lambda-H^{\prime} X\right)\right|<\alpha_{4}\left(L^{j} \eta\right)^{-1} \quad \text { on } \quad \Omega_{j},
$$

and by the inequality (214) we have

$$
\left|C^{\prime}\left(\lambda-H^{\prime} X\right)\right|<C_{2}^{\prime}\left(\alpha_{3}+\alpha_{4}\right) \alpha_{4},
$$

where the constant $O(1)$ in (214) [3] was denoted by $C_{2}^{\prime}$. The transformation (1.118) maps the set (1.119) of $X$ 's into itself if

$$
C_{2}^{\prime}\left(\alpha_{3}+\alpha_{4}\right) \alpha_{4} \leqq \frac{1}{2 B_{0}^{\prime}} \alpha_{4}, \quad \text { or } \quad \alpha_{3}+\alpha_{4} \leqq \frac{1}{2 B_{0}^{\prime} C_{2}^{\prime}} .
$$

We may admit configurations $\lambda, X$ with values in the complexified algebra $\mathfrak{g}^{c}$ and all the above equations and inequalities are valid also.

We will prove that the mapping (1.118) is a contraction on the set (1.119) for $\alpha_{3}$, $\alpha_{4}$ sufficiently small. We have

$$
\begin{gathered}
C^{\prime}\left(\lambda-H^{\prime} X_{1}\right)-C^{\prime}\left(\lambda-H^{\prime} X_{2}\right)=\int_{0}^{1} d t \frac{d}{d t} C^{\prime}\left(\lambda-H^{\prime}\left(t X_{1}+(1-t) X_{2}\right)\right) \\
=-\int_{0}^{1} d t\left\langle\frac{\delta}{\delta \lambda} C^{\prime}\left(\lambda-H^{\prime}\left(t X_{1}+(1-t) X_{2}\right)\right), H^{\prime}\left(X_{1}-X_{2}\right)\right\rangle,
\end{gathered}
$$

where the functional derivative $\frac{\delta}{\delta \lambda} C^{\prime}(\lambda)$ is defined as the linear mapping

$$
\left\langle\frac{\delta}{\delta \lambda} C^{\prime}(\lambda), \lambda_{0}\right\rangle=\left.\frac{d}{d \tau} C^{\prime}\left(\lambda+\tau \lambda_{0}\right)\right|_{\tau=0} .
$$


Using the analyticity properties of $C^{\prime}(\lambda)$, the derivative above can be written as

$$
\left\langle\frac{\delta}{\delta \lambda} C^{\prime}(\lambda), \lambda_{0}\right\rangle=\frac{1}{2 \pi i} \int_{|\tau|=r} d \tau \frac{1}{\tau^{2}} C^{\prime}\left(\lambda+\tau \lambda_{0}\right) .
$$

Taking $r=\left(2 \max \left\{\left|\lambda_{0}\right|,\left|D \lambda_{0}\right|\right\}\right)^{-1} \alpha_{4}$, we get the estimate

$$
\left|\left\langle\frac{\delta}{\delta \lambda} C^{\prime}(\lambda), \lambda_{0}\right\rangle\right| \leqq C_{2}^{\prime} 2 \max \left\{\left|\lambda_{0}\right|,\left|D \lambda_{0}\right|\right\}\left(\alpha_{3}+\alpha_{4}\right) .
$$

Applying it to the expression (1.122) we have

$$
\left|C^{\prime}\left(\lambda-H^{\prime} X_{1}\right)-C^{\prime}\left(\lambda-H^{\prime} X_{2}\right)\right| \leqq C_{2}^{\prime} 2 B_{0}^{\prime}\left(\alpha_{3}+\alpha_{4}\right)\left|X_{1}-X_{2}\right|,
$$

hence the mapping (1.118) is contractive if e.g. $\alpha_{3}+\alpha_{4} \leqq \frac{1}{4 B_{0}^{\prime} C_{2}^{\prime}}$. If the last condition is satisfied, then the mapping transforms the set $\left\{|X|<\frac{1}{2 B_{0}^{\prime}} \alpha_{4}\right\}$ into itself and is contractive on this set. Thus by the contraction mapping theorem there exists exactly one solution of Eq. (1.117). This solution is an analytic function of $\lambda$ defined on the set of $\lambda$ satisfying (1.119). We take $D^{\prime}(\lambda)$ equal to this solution. From Eq. (1.116) we can get much better bounds on it:

$$
\left|D^{\prime}(\lambda)\right|=\left|C^{\prime}\left(\lambda-H^{\prime} D^{\prime}(\lambda)\right)\right|<C_{2}^{\prime}\left(\alpha_{3}+\alpha_{4}\right) \alpha_{4} .
$$

They imply in particular that the mapping (1.113) transforms the set $\left\{\lambda:|\lambda|<\frac{1}{2} \alpha_{4}\right.$, $|D \lambda|<\frac{1}{2} \alpha_{4}\left(L^{j} \eta\right)^{-1}$ on $\left.\Omega_{j}\right\}$ onto a set containing $\left\{\lambda^{\prime}:\left|\lambda^{\prime}\right|<\frac{1}{4} \alpha_{4},\left|D \lambda^{\prime}\right|<\frac{1}{4} \alpha_{4}\left(L^{j} \eta\right)^{-1}\right.$ on $\left.\Omega_{j}\right\}$ for $\alpha_{3}, \alpha_{4}$ sufficiently small.

\section{F. Removal of the Additional Regularity Assumption}

We will prove that the additional regularity assumption (3.35) in (1.33) is a consequence of the fundamental assumption $U_{0} \in \mathfrak{U}_{k}\left(\left\{\Omega_{j}\right\}, \alpha_{0}\right)$, thus of the regularity conditions (1.7)-(1.9). In fact we will prove a more general, and much more precise, theorem which will have other important applications in subsequent papers.

At first let us notice that if $\alpha_{0}+\alpha_{1} \leqq c_{1}$ and

$$
U_{0} \in \mathfrak{H}_{k}\left(\left\{\Omega_{j}\right\}, \alpha_{0}\right),\left|\bar{U}_{0}^{j}-1\right|<\alpha_{1} \quad \text { on } \quad \Lambda_{j}, j=0,1, \ldots, k,
$$

then Theorem 2 implies that there exists a gauge transformation $u$ such that

$$
U_{0}^{u^{-1}}=e^{i \eta A},|A|,\left|\nabla^{\eta} A\right|<B_{1}\left(\alpha_{0}+\alpha_{1}\right)\left[\left(L^{j} \eta\right)^{-1},\left(L^{j} \eta\right)^{-2}\right] \quad \text { on } \Omega_{j}, j=0,1, \ldots, k .
$$

We have to transform $U_{0}$ into a configuration satisfying the axial gauge conditions (1.15), i.e. we get a configuration belonging to $A x_{k}\left(\mathfrak{B}_{k}, 1\right)$. It is achieved by applying a gauge transformation $u_{0}$ satisfying the conditions $u_{0}(y)=1$ for $y \in \mathfrak{B}_{k}$, hence the configuration $U_{0}^{u} \overline{0}^{-1}$ satisfies (1.126). Then we apply Theorem 2 to the configurations $U_{0}^{u{ }^{1}}, 1$ in the place of $U^{\prime} U_{0}, U_{0}$, and we get (1.127). The configuration 
identically equal to 1 satisfies, of course, all possible regularity conditions. Let us notice that the gradient $\nabla^{\eta} A$ in (1.127) is the ordinary finite difference gradient. Of course (1.127) implies the regularity condition (3.35). We will apply this remark to specially chosen $\left\{\Omega_{j}\right\}$.

Now let us take a cube $\square \subset \Omega_{0}$ for which we want to prove the condition (3.35). A size of this cube depends on an index $j$ indicating a scale we are interested in. We assume that the cube $\square$ is contained in $\Omega_{j}$, and not in $\Omega_{j+1}$. Further, we assume that it is a union of cubes of the size $R_{1} M_{1} L^{j} \eta$, where $R_{1}, M_{1}$ are smallest integers for which all the theorems of the papers $[2,4]$ are valid. To prove condition (3.35) it is enough to take one such cube. Having in view future applications we take a size of $\square$ equal to $M L^{j} \eta$, where $M$ is a multiple of $R_{1} M_{1}$. Without a loss of generality we can assume also that $j=k$, because we can drop out the domains $\Omega_{j^{\prime}}, j^{\prime}>j$, from our assumptions, and change a scale. Then we have to admit the value $k=0$. In this case the reasoning is much simpler and will be covered by our analysis of a general case, so we can assume that $k \geqq 1$.

Let us take a sequence of cubes $\square_{0}, \square_{1}, \ldots, \square_{k-1}, \square_{k}, \square$, such that $\square_{j} \supset \square_{j+1}$ and a distance between boundaries of these cubes is equal to $R_{1} M_{1} L^{j} \eta$. Thus a distance of the boundary of $\square_{0}$ to $\square$ is equal to

$$
\sum_{j=0}^{k} R_{1} M_{1} L^{j} \eta<\frac{1}{1-L^{-1}} R_{1} M_{1} \leqq 2 R_{1} M_{1}
$$

We cover $\square_{0}$ by a smallest family of cubes of the size $R_{1} M_{1}$. A sum of these cubes is a cube which we denote by $\tilde{\square}$. A distance of its boundary to $\square$ is equal to $2 R_{1} M_{1}$. We assume that $R M$ is bigger than $R_{1} M_{1}$, for example $L^{-1} R M>2 d R_{1} M_{1}$, thus we have $\tilde{\square} \subset \Omega_{k-1}$. Of course we assume also that these constructions are compatible with the block structure of the lattice $T$, i.e. for every $j$ the cube $\square_{j}$ is a sum of the big blocks of the lattice $T_{L^{-j}}$.

Let us consider the configuration $U_{0}$ on the cube $\tilde{\square}$. By the assumptions (1.7)-(1.9) and Proposition 2 from [3] we have

$$
\left|\bar{U}_{0}^{j}(\partial p)-1\right|<2 \alpha_{0} L^{2}\left(L^{j} \eta\right)^{2}, \quad p \subset \tilde{\square}^{(j)}, \quad j=0,1, \ldots, k .
$$

We apply a gauge transformation to $U_{0}$, such that the gauge transformed configuration $U_{0}^{\prime}$ satisfies the axial gauge conditions (1.15), and $\bar{U}_{0}^{\prime k}$ satisfies the global axial gauge conditions on $\tilde{\square}^{(k)}$ of the type introduced in the proof of Proposition 1 in [3], i.e. $\bar{U}_{0}^{\prime k}\left(\Gamma_{y, x}\right)=1$ for $x \in \tilde{\square}^{(k)}$, where $y$ is a center of $\tilde{\square}^{(k)}$. These last conditions imply

$$
\left|\bar{U}_{0}^{\prime k}\left(x, x^{\prime}\right)-1\right|<|x-y| 2 L^{2} \alpha_{0} \leqq\left(M+4 R_{1} M_{1}\right) d L^{2} \alpha_{0},\left\langle x, x^{\prime}\right\rangle \subset \tilde{\square}^{(k)} .
$$

We assume that $\alpha_{0}$ is so small that the number on the right-hand side above is still small and all the theorems on averaging operations are valid. In particular this means $\alpha_{0} \leqq O(1) M^{-1}$, where $O(1)$ has to be a small number. If we are proving the condition (3.35), then $M=R_{1} M_{1}$ and the restriction is $\alpha_{0} \leqq O(1)\left(R_{1} M_{1}\right)^{-1} . R_{1}$, $M_{1}$ are absolute constants, hence the last restriction is of the same kind as the other restrictions on $\alpha_{0}$. Applying Lemma 1 many times, as in the proof of (1.65), (1.66), 
we get

$$
\begin{aligned}
& \left|\bar{U}_{0}^{\prime j}\left(x, x^{\prime}\right)-1\right|<8 d^{2} L^{2}\left(L^{-2(k-j-1)}+\ldots+L^{-2}+1\right) \alpha_{0} \\
& \quad+\left(M+4 R_{1} M_{1}\right) d L^{2} \alpha_{0}<11 d^{2} L^{2} \alpha_{0}+5 d L^{2} M \alpha_{0}<6 d L^{2} M \alpha_{0},\left\langle x, x^{\prime}\right\rangle \subset \tilde{\square}^{(j)} .
\end{aligned}
$$

The sequence of cubes $\left\{\square_{j}\right\}$ is an admissible family of subsets satisfying (1.3), (1.4), $\square_{k} \subset \Omega_{k-1}, \square_{j} \subset \Omega_{j}, j<k$. Let us define

$$
\begin{aligned}
& \Lambda_{j}^{\prime}=\square_{j}^{(j)} \backslash \square_{j+1}^{(j)}, j=1, \ldots, k-1, \Lambda_{k}^{\prime}=\square_{k}^{(k)}, \Lambda_{0}^{\prime}=T \backslash \square_{1}, \\
& \mathfrak{C}_{k}=\bigcup_{j=0}^{k} \Lambda_{j}^{\prime},
\end{aligned}
$$

and let us define a configuration $U_{0}^{\prime \prime}$ as equal to $U_{0}^{\prime}$ on $\tilde{\square}$, and equal to 1 outside $\tilde{\square}$. It satisfies the conditions

$$
\begin{gathered}
U_{0}^{\prime \prime} \in \mathfrak{U}_{k}\left(\left\{\square_{j}\right\}, L^{3} \alpha_{0}\right) \cap A x_{k}\left(\mathfrak{C}_{k}, 1\right), \\
\left|\overline{U_{0}^{\prime \prime} j}-1\right|<6 d L^{2} M \alpha_{0} \quad \text { on } \quad \square_{j}^{(j)}, \quad j=0,1, \ldots, k,
\end{gathered}
$$

by the construction of $U_{0}^{\prime \prime}$, and the inequality (1.130). If $7 d L^{2} M \alpha_{0} \leqq c_{1}$, then the assumptions of Theorem 4 are satisfied for the pair of configurations $1, U_{0}^{\prime \prime}$, thus there exists a gauge transformation $u$ such that $U_{1}=U_{0}^{\prime \prime u^{-1}}$ satisfies the conditions (1.36)-(1.39). Especially we have for $M=R_{1} M_{1}$,

$$
U_{1}=e^{i \eta A},|A|,\left|\nabla^{\eta} A\right|<7 d L^{2} B_{1} R_{1} M_{1} \alpha_{0} \quad \text { on } \square .
$$

The configuration $U_{1}$ in a neighborhood of $\square$ is obtained from $U_{0}$ by a gauge transformation, hence for $\alpha_{0}$ sufficiently small we have proved the regularity condition (3.35).

This implies that we can drop out this condition from the assumption (1.33).

We formulate the more general result in

Proposition 6. Let $U_{0}, U_{0}^{\prime}, \square, \tilde{\square}$ be as described above, and let $7 d L^{2} M \alpha_{0} \leqq c_{1}$. There exists a gauge transformation $u$ defined on $\tilde{\square}$ and such, that

$$
\begin{gathered}
U_{0}^{u^{-1}}=U_{1}=e^{i \eta A} \quad \text { on } \tilde{\square}, \\
L^{j} \eta|A|,\left(L^{j} \eta\right)^{2}\left|\nabla^{\eta} A\right|,\left(L^{j} \eta\right)^{3}\left|\partial^{\eta *} \partial^{\eta} A\right|,\left(L^{j} \eta\right)^{3}\left|\Delta^{\eta} A\right|<7 d L^{2} B_{1} M \alpha_{0} \quad \text { on } \square_{j}, \\
Q_{k}(\eta A)=\frac{1}{i} \log \overline{U_{0}^{\prime} k} \text { on } \square^{(k)},\left|\frac{1}{i} \log \overline{U_{0}^{\prime}} k\left(x, x^{\prime}\right)\right|<|x-y| 4 \alpha_{0} \leqq 2 d M \alpha_{0} \\
\text { for }\left\langle x, x^{\prime}\right\rangle \subset \square^{(k)}, y \text { is a center of } \square, \\
R \partial^{\eta *} A=0 \text {, the operator } R \text { is determined by }\left\{\square_{j}\right\} .
\end{gathered}
$$

The functions and derivatives above are defined without any external gauge field configuration (or the configuration is equal to 1). 


\section{G. An Inverse Theorem to Theorem 2}

In this section we will prove a theorem which is, in a certain sense, inverse to Theorem 2. We assume that we are given a gauge field configuration $U_{0}$,

$$
U_{0} \in \mathfrak{U}_{k}\left(\left\{\Omega_{j}\right\}, \alpha_{0}\right),
$$

and a Lie algebra valued configuration $A$ satisfying

$$
L^{j} \eta|A|,\left(L^{j} \eta\right)^{2}\left|\nabla_{U_{0}}^{\eta} A\right|,\left(L^{j} \eta\right)^{3}\left|D_{U_{0}}^{\eta *} D_{U_{0}}^{\eta} A\right|<\alpha_{2} \quad \text { on } \quad \Omega_{j} .
$$

We consider the configuration $U_{1} U_{0}, U_{1}=e^{i \eta A}$. We would like to prove bounds (1.7) (1.9), i.e. to find spaces $\mathfrak{U}_{k}\left(\left\{\Omega_{j}\right\}, \alpha\right)$ to which this configuration belongs.

Let us start with estimates of $\partial U_{1} U_{0}-1$. The identity (1.21), the bound (1.47) and the assumptions (1.139), (1.140) imply

$$
\begin{aligned}
\left|\left(U_{1} U_{0}\right)(\partial p)-1\right| & \leqq\left|U_{0}(\partial p)-1\right|+\eta^{2}\left|\left(D_{U_{0}}^{\eta} A\right)(p)\right|+\frac{1}{2} \eta^{2}(\partial|A|(p))^{2} \\
& <\left(\alpha_{0}+2 \alpha_{2}+8 \alpha_{2}^{2}\right) L^{-2 j} \text { on } \Omega_{j} .
\end{aligned}
$$

The basic estimate (1.54) and the assumptions imply

$$
\left|D_{U_{1} U_{0}}^{\eta *} \partial U_{1} U_{0}\right|<\left(\alpha_{0}+\alpha_{2}+36 d \alpha_{2}^{2}+50 d \alpha_{2}^{3}+10 d \alpha_{0} \alpha_{2}\right)\left(L^{j} \eta\right)^{-3} \eta^{2} .
$$

To simplify formulations we assume that $\alpha_{0}, \alpha_{2}$ are so small that $\alpha_{0}+2 \alpha_{2}+8 \alpha_{2}^{2}$ $\leqq \alpha_{0}+3 \alpha_{2}, \alpha_{0}+\alpha_{2}+36 d \alpha_{2}^{2}+50 d \alpha_{2}^{3}+10 d \alpha_{0} \alpha_{2} \leqq \alpha_{0}+2 \alpha_{2}$. The Proposition 4 from [3] gives also

$$
\left|Q_{j}\left(U_{0}, \eta A\right)\right|<2 \alpha_{2} \text { on } \Omega_{j}^{(j)} .
$$

Let us take a gauge transformation $u$ satisfying the conditions (1.29) and such that the configuration $U^{\prime}=\left(U_{1} U_{0}\right)^{u} U_{0}^{-1}$ satisfies the axial gauge conditions (1.19). This gauge transformation is determined uniquely. The above bounds imply the following

Proposition 7. If the configurations $U_{0}, A$ satisfy (1.139), (1.140), then for $\alpha_{0}, \alpha_{2}$ sufficiently small we have

$$
\begin{gathered}
U^{\prime} U_{0}=\left(U_{1} U_{0}\right)^{u} \in \mathfrak{H}_{k}\left(\left\{\Omega_{j}\right\}, \alpha_{0}+3 \alpha_{2}\right) \cap A x_{k}\left(\mathfrak{B}_{k}, U_{0}\right), \\
\left.\mid \overline{\left(U^{\prime} U_{0}\right.}\right)^{j}-\bar{U}_{0}^{j}|=| \exp i Q_{j}\left(U_{0}, \eta A\right)-1 \mid<2 \alpha_{2} \text { on } \Omega_{j}^{(j)} .
\end{gathered}
$$

This theorem complements Theorem 2. The gauge transformations constructed there establish not only a mapping of the space (1.33)-(1.35) into the space (1.36)-(1.39), but a kind of isomorphism also, in the sense that the inverse mapping transforms the second space, defined by some constant $\alpha_{2}$ instead of $B_{1}\left(\alpha_{0}+\alpha_{1}\right)$, into the first one with properly chosen $\alpha_{0}, \alpha_{1}$.

\section{H. Generalizations of the Main Results}

Let us describe two possible generalizations. The first is connected with the fundamental gauge fixing condition (1.38) in the formulations of Theorems 2, 4. It is written with 0 on the right-hand side, but nothing prevents us to consider a more 
general condition of the form

$$
R\left(U_{0}\right) D_{U_{0}}^{\eta *} A=f,
$$

where $f$ is a function from the space $R\left(U_{0}\right)$, i.e. a Lie algebra valued function defined on $\Omega_{0}$ and satisfying $R\left(U_{0}\right) f=f$. Of course we have to assume that $f$ is in a sufficiently small neighborhood of 0 , for example it is enough to assume that $|f|_{(-2)}<\gamma\left(\alpha_{0}+\alpha_{1}\right)$ with a positive, not too big, constant $\gamma$, e.g. $\gamma=1$. Inspecting the proofs of the theorems and propositions we can see easily that they work in this more general situation almost without any changes, only some constants change their numerical values. Thus we have the following generalization of Theorem 2.

Theorem 8. There exist constants $B_{1}, B_{2}\left(\beta_{0}\right), c_{1}$ such that for arbitrary $U_{0}, U^{\prime} U_{0}$ satisfying (1.33)-(1.35) with $\alpha_{0}+\alpha_{1} \leqq c_{1}$, and for an arbitrary function $f$ from the space $R\left(U_{0}\right)$ satisfying the bound $|f|_{(-2)}<\gamma\left(\alpha_{0}+\alpha_{1}\right)$, there exists exactly one gauge transformation $u$ satisfying (1.29) and such, that the conditions (1.36), (1.37), (1.39), and (1.146) hold for the configuration $U_{1}=U^{u^{-1}}$. The constants $B_{1}, B_{2}\left(\beta_{0}\right)$ are as in Theorems 2, 4, the constant $c_{1}$ depends on $d, L$ and $\gamma$.

At one point in the next paper we will use this theorem.

A second generalization is connected with the second regularity condition (1.9). It is a strong assumption, and although it is a natural one because it is satisfied by solutions of the variational problem described in [1], it would be desirable to have a more general condition, and a more general result on Landau gauges. In fact it is possible to have such a generalization. Instead of condition (1.9), we assume the following, weaker Hölder regularity condition,

$$
\frac{1}{\left|x-x^{\prime}\right|^{\beta_{0}}}\left|R\left(U\left(\Gamma_{x, x^{\prime}}\right)\right) U\left(\partial p_{\mu v}\left(x^{\prime}\right)\right)-U\left(\partial p_{\mu v}(x)\right)\right|<\alpha_{0} L^{-2 j}\left(L^{j} \eta\right)^{-\beta_{0}}
$$

for

$$
x, x^{\prime} \in \Omega_{j},\left(L^{j} \eta\right)^{-1}\left|x-x^{\prime}\right| \leqq 1,1 \leqq \mu<v \leqq d, \quad j=0,1, \ldots, k,
$$

and for some positive $\beta_{0}, 0<\beta_{0} \leqq 1$. With these conditions we can prove again an analog of Theorem 2 , the only difference is that the configuration $U_{1}$, or rather $A=\frac{1}{i \eta} \log U_{1}$, satisfies conditions (1.36)-(1.38), with $\beta<\beta_{0}$, but of course not condition (1.39). Unfortunately a proof of this result is rather unelementary, and although it follows the main line of arguments of this paper, it makes use of operators, e.g. $G^{\prime \beta}\left(U_{0}\right)$, and their properties. It would be necessary to extend in an essential way our theory of Green's functions in [4], to include necessary results. We will not need the above generalization, so let us stop the discussion mentioning only the possibility of such results.

\section{References}

1. Bałaban, T.: Renormalization group methods in non-Abelian gauge theories. Harvard preprint, HUTMP B134

2. Bałaban, T.: Propagators and renormalization transformations for lattice gauge theories, I, II. Commun. Math. Phys. 95, 17-40 (1984) and 96, 223-250 (1984) 
3. Bałaban, T.: Averaging operations for lattice gauge theories. Harvard preprint, Commun. Math. Phys. (to appear)

4. Bałaban, T.: Propagators for lattice gauge theories in a background field. Commun. Math. Phys. (to appear)

5. Brydges, D., Fröhlich, J., Seiler, E.: On the construction of quantized gauge fields. I. General results. Ann. Phys. 121, 227-284 (1979)

6. Osterwalder, K., Seiler, E.: Gauge field theories on a lattice. Ann. Phys. 110, 440-471 (1978)

7. Wilson, K.G.: Quantum chromodynamics on a lattice. In: Quantum field theory and statistical mechanics, Cargèse 1976, pp. 143-172. Lévy, M., Mitter, P. (eds.). New York: Plenum Press 1977

Communicated by A. Jaffe

Received August 9, 1984; in revised form December 20, 1984 